\title{
DISCRETE TIME OPTIMAL CONTROL WITH FREQUENCY CONSTRAINTS FOR NON-SMOOTH SYSTEMS
}

\author{
SHRUTI KOTPALLIWAR, PRADYUMNA PARUCHURI, DEBASISH CHATTERJEE, \\ AND RAVI BANAVAR
}

March 28, 2019

\begin{abstract}
We present a Pontryagin maximum principle for discrete time optimal control problems with (a) pointwise constraints on the control actions and the states, (b) smooth frequency constraints on the control and the state trajectories, and (c) nonsmooth dynamical systems. Pointwise constraints on the states and the control actions represent desired and/or physical limitations on the states and the control values; such constraints are important and are widely present in the optimal control literature. Constraints of the type (b), while less standard in the literature, effectively serve the purpose of describing important properties of inertial actuators and systems. The conjunction of constraints of the type (a) and (b) is a relatively new phenomenon in optimal control but are important for the synthesis control trajectories with a high degree of fidelity. The maximum principle established here provides first order necessary conditions for optimality that serve as a starting point for the synthesis of control trajectories corresponding to a large class of constrained motion planning problems that have high accuracy in a computationally tractable fashion. Moreover, the ability to handle a reasonably large class of nonsmooth dynamical systems that arise in practice ensures broad applicability of our theory, and we include several illustrations of our results on standard problems.
\end{abstract}

\section{InTRODUCTION}

Optimal control theory, arguably, started in the avatar of the Brachystochrone problem of J. Bernoulli in the late 17th century [SW97], and over the intervening centuries has evolved into a subject that offers a powerful set of tools for control synthesis. Especially relevant in the context of practical applications are synthesis techniques that seamlessly integrate constraints on the states and control actions while maintaining computational tractability. The literature on such constrained problems is certainly not as vast as the counterpart for unconstrained problems, and our article contributes to precisely this body of work.

Under the overarching stipulation of computationally tractable control synthesis techniques, there are two specific contributions of our work. The first concerns the simultaneous inclusion of the following four different classes of constraints in control problems:

- constraints on the control actions pointwise in time,

- constraints on the states pointwise in time,

- frequency constraints on the control trajectories, and

$\circ$ frequency constraints on the state trajectories.

Key words and phrases. frequency constraints, optimal control, Pontryagin maximum principle, nonsmooth systems.

The authors were supported partially by the grant 17ISROC001 from the Indian Space Research Organization. They thank Soumitro Banerjee for useful pointers to the literature on control of power electronic circuits, and Karmvir Singh Phogat for helpful discussions and access to his software. 
Each of these four types of constraints is extremely important in practical applications. Actuators are governed by the laws of physics and cannot deliver signals with magnitudes that are beyond their physical limitations; this naturally means that the set of admissible control actions is constrained. Controlled dynamical systems are typically permitted to operate only within reasonable boundaries to avoid fatigue, premature ageing and disintegration of their components; this naturally imposes constraints on their states. Frequency constraints on the control trajectories are needed for all inertial actuators, without which the synthesized control signals may contain frequencies that cannot be faithfully reproduced by the actuators due to their physical limitations; in such cases, differences arise between the predicted and observed behaviours, leading to loss of precision in their desired performances. To take care of this issue, frequently in practice, the synthesized controls are passed through a filter located before the actuator to ensure satisfaction of the desired spectrum. This procedure, however, distorts the original signal that was designed with the desired performance objectives in mind, and hence the system performance deteriorates. Frequency constraints on the state trajectories are relevant in inertial controlled systems: they are especially useful to prevent undesirable vibrations of flexible structures in mechanical objects such as satellites, aeroplanes, robotic arms, or to induce desirable damping in flexible structures, etc., and vibration control has been an active area of control for the past several decades.

On the one hand, the first two types of constraints have been studied extensively in the context of optimal control, and are, to a fair extent, amenable to computationally constructive synthesis techniques. Indeed, control action constraints are common place in optimal control theory: both the Pontryagin maximum principle and dynamic programming techniques [IT09, Lib11, Dub78, DM65, Ber12] permit the inclusion of control action constraints pointwise in time. While dynamic programming is not always computationally tractable (e.g., for high-dimensional systems), there are numerical algorithms that employ the Pontryagin maximum principle to synthesize constrained optimal control trajectories. Algorithmic techniques relying on viability theory [ABSP11], while computationally demanding (to the point of being prohibitive for high-dimensional systems), permit the inclusion of state constraints pointwise in time in addition to control constraints. On the other hand, the inclusion of frequency constraints on the control and state trajectories is relatively uncommon in the literature. Despite the fact that the classical feedback techniques of continuous-time $H_{\infty}$ control [DFT92] do deal with frequency domain behaviour of the control trajectories and are capable of indirectly realizing restrictions on frequency components via penalization of certain bands, neither sharp cut-offs in the spectrum nor control and state constraints can be readily enforced via this technique. Frequency constraints on the state trajectories, especially in the context of nonlinear systems, have been treated sparsely in the literature; typical existing approaches rely on frequency domain techniques that are ill-suited to handle state and control constraints and are difficult to apply to nonlinear systems. In fact, apart from the US Patent [SB95], we are unaware of extensive studies that impose frequency constraints on the states and control trajectories. In particular, our own previous studies [PC19, $\left.\mathrm{KPP}^{+} 18\right]$ have only focussed on frequency constraints on the control trajectories, and the simultaneous inclusion of time and frequency constraints with precise bounds and cut-offs on frequency bands are not to be found in the existing literature to the best of our knowledge. Our current results address these lacunae: we establish a version of the Pontryagin maximum principle for optimal control of discrete time nonlinear control systems for which the aforementioned constraints are all enforced simultaneously. These constraints are specifically illustrated in $\$ 6.1$ on a linear approximation of an inverted pendulum on a cart around the unstable equilibrium position of the pendulum. We specifically impose state constraints so that the validity of the linearization is maintained, and demonstrate that simple point-to-point manoeuvres are dramatically altered by an ad-hoc posteriori application of frequency filters. 
The second specific contribution of this article is the ability to encompass nonsmooth problem data. Nonsmooth dynamical systems arise naturally in a variety of application areas [AB08], including those where the physical characteristics of devices involve nonlinearities such as the norm instead of its square [GP17], or when the dynamical equations are defined piece-wise over disjoint domains of the state-space as a result of intrinsic properties of devices [BKYY00]. The Pontryagin maximum principle established in this article, although not catering to the most general situation insofar as nonsmoothness is concerned, handles a large class of nonsmooth nonlinearities that arise in practical applications. ${ }^{1}$ This specific aspect of our results is illustrated on two examples in $\S 6$ : a standard non-smooth dynamical system and the popular buck converter power electronic circuit.

The optimal control synthesis procedure presented in this article proceeds with models in discrete time. We presume that these discrete time models faithfully represent the dynamics of the system, and do not concern ourselves here with the issues of discretization of continuous-time systems. Our preference for discrete time models is motivated by two factors, the first of which is the observation that the final implementation of controllers are carried out digitally via sample-and-hold mechanisms, and therefore, naturally involves a time discretization. The other factor is essentially that of convenience: the mathematical and numerical complexity involved in incorporating the four types of constraints that we want in the continuous time is formidable if not outright impossible.

This article unfolds as follows: We provide in $\S 2$ a precise mathematical statement of our optimal problem that incorporates pointwise constraints on the states and the control actions, and frequency constraints on the control and the state trajectories, for a large class of nonsmooth dynamical systems. $\S 3$ consist of a set of preliminaries needed for the proof of main result. In $\S 4$ we establish a set of first order necessary conditions for optimality in the optimal control problem defined in $\$ 2$; this is the central contribution of this article. We follow up with some technical remarks and immediate corollaries. $\S 5$ is devoted for the detailed proof of main result, and in the final $\S 6$ we present several examples to illustrate the proposed necessary conditions. The appendices $\S \mathrm{A}$ and $\S \mathrm{B}$ consist of several auxiliary results that are employed in the proof of our main result.

\section{Problem Setup}

Consider a discrete time control system whose dynamics is governed by the difference equation

$$
x_{t+1}=f_{t}\left(x_{t}, u_{t}\right) \text { for } t=0, \ldots, N-1,
$$

with the following data:

(2.1-a) $x_{t} \in \mathbb{R}^{d}$ is the vector of state at time $t$,

(2.1-b) $u_{t} \in \mathbb{R}^{m}$ is the vector of control action at time $t$,

(2.1-c) $\mathbb{R}^{d} \times \mathbb{R}^{m} \ni(\xi, \mu) \longmapsto f_{t}(\xi, \mu) \in \mathbb{R}^{d}$ for $t=0, \ldots, N-1$, is a given family of locally Lipschitz continuous maps.

In this article we derive first order necessary conditions - a Pontryagin Maximum Principle - for discrete time optimal control problems with nonsmooth dynamics and frequency constraints on the state and control trajectories in addition to the standard constraints on the control magnitudes and the states. The constraints on the frequencies of the states and control trajectories refer to constraints on the discrete Fourier transform(DFTs) of the individual components of the states and controls. Constraints on the frequency spectra of the control trajectories appeared in the [PC19]; here we move one step further by permitting constraints on the spectra of the state trajectories, to be present as a given stipulation.

\footnotetext{
${ }^{1}$ See also the discussion immediately preceding (2.2) concerning possible generalizations.
} 
In addition to handling frequency constraints, our result also encompasses piecewise smooth dynamical system such as the ones mentioned in $\S 1$, and accordingly, we permit our dynamics to be nonsmooth. A more general framework where both the cost-per-stage and the dynamics are nonsmooth, is possible, and such a general framework is typically employed to compare the necessary conditions for a discrete time optimal control problem and a discrete time approximation of a continuous time problem; see, e.g., [Mor06b]. We do not aim for maximal generality in our work primarily to ensure a clean calculus; in fact, some of our assumptions are aimed at simplifying the necessary conditions that arise in more general situations.

Here is the precise mathematical statement of our problem:

\begin{tabular}{|c|c|}
\hline$\underset{\left(u_{t}\right)_{t=0}^{N-1}}{\operatorname{minimize}}$ & $\sum_{t=0}^{N-1} c_{t}\left(x_{t}, u_{t}\right)+c_{N}\left(x_{N}\right)$ \\
\hline subject to & $\left\{\begin{array}{l}\text { dynamics }(2.1), \\
x_{t} \in \mathbb{S}_{t} \quad \text { for } t=0, \ldots, N, \\
u_{t} \in \mathbb{U}_{t} \quad \text { for } t=0, \ldots, N-1, \\
F\left(x_{0}, \ldots, x_{N}\right)=0, \\
G\left(u_{0}, \ldots, u_{T-1}\right)=0,\end{array}\right.$ \\
\hline
\end{tabular}

where

(2.2-a) the mapping $\mathbb{R}^{d} \times \mathbb{R}^{m} \ni(\xi, \mu) \longmapsto c_{t}(\xi, \mu) \in \mathbb{R}$ for each $t=0, \ldots, N-1$, is continuously differentiable and defines a sequence of cost per stage functions, and $\mathbb{R}^{d} \ni \xi \longmapsto c_{N}(\xi) \in \mathbb{R}$ is a continuously differentiable final stage cost,

(2.2-b) $\left(\mathbb{S}_{t}\right)_{t=0}^{N}$ is a sequence of closed subsets of $\mathbb{R}^{d}$ describing a tube (over time) of admissible states,

(2.2-c) $\left(\mathbb{U}_{t}\right)_{t=0}^{N-1}$ is a sequence of closed subsets of $\mathbb{R}^{m}$ depicting the sets of admissible control actions at each time $t$,

(2.2-d) given $v_{x} \in \mathbb{N}$, some vector $\Upsilon_{x} \in \mathbb{R}^{v_{x}}$, and smooth functions $\widetilde{F}_{t}: \mathbb{R}^{d} \rightarrow \mathbb{R}^{v_{x}}$ for $t=0, \ldots, N$, we define the map $\mathbb{R}^{d(N+1)} \ni\left(\xi_{0}, \ldots, \xi_{N}\right) \longmapsto F\left(\xi_{0}, \ldots, \xi_{N}\right):=$ $\Upsilon_{x}+\sum_{t=0}^{N} \widetilde{F}_{t}\left(\xi_{t}\right) \in \mathbb{R}^{v_{x}}$ (this map $F$ will be employed to quantify constraints on the frequency spectra of the state trajectories),

(2.2-e) given $v_{u} \in \mathbb{N}$, some vector $\Upsilon_{u} \in \mathbb{R}^{v_{u}}$, and smooth functions $\widetilde{G}_{t}: \mathbb{R}^{m} \rightarrow$ $\mathbb{R}^{v_{u}}$ for $t=0, \ldots, N-1$, we define the map $\mathbb{R}^{m N} \ni\left(\mu_{0}, \ldots, \mu_{N-1}\right) \longmapsto$ $G\left(\mu_{0}, \ldots, \mu_{N-1}\right):=\Upsilon_{u}+\sum_{t=0}^{N-1} \widetilde{G}_{t}\left(\mu_{t}\right) \in \mathbb{R}^{v_{u}}$ (this map $G$ will be employed to quantify constraints on the frequency spectra of the control trajectories).

Remark 2.1. For the control trajectory $\left(u_{t}\right)_{t=0}^{N-1}$, let $u^{(k)}:=\left(u_{t}^{(k)}\right)_{t=0}^{N-1}$ denote the trajectory of the $k^{\text {th }}$ component of the control for each $k=1, \ldots, m$. For each component trajectory $u^{(k)}$, we obtain the vector of DFT coefficients $\widehat{u^{(k)}}:=\mathbf{F} u^{(k)} \in \mathbb{C}^{N}$ where $\mathbf{F} \in \mathbb{C}^{N \times N}$ denotes the DFT matrix corresponding to signal length $N$ (see [SS03, Chapter 7]). Let $\widehat{u}:=$

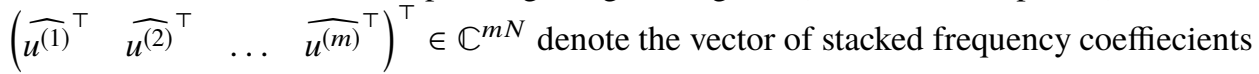
of all components $u^{(k)}, k=1, \ldots, m$. That is,

$$
\mathbb{C}^{m N} \ni \widehat{u}:=\left(\begin{array}{c}
\widehat{u^{(1)}} \\
\vdots \\
\widehat{u^{(m)}}
\end{array}\right)=\left(\begin{array}{c}
\mathbf{F} u^{(1)} \\
\vdots \\
\mathbf{F} u^{(m)}
\end{array}\right)
$$

By frequency constraints on the control trajectory, we refer to constraints on the DFT coefficients of the trajectories of the components of control. We specialize our results in this article to constraints that eliminate certain frequency components of the control trajectories, 
i.e., restrict certain entries of $\widehat{u}$ to zero. By carefully choosing the rows corresponding to the desired frequency components to be restricted, separating the real and imaginary parts, and using a suitable rearrangement, one obtains constraints of the form mentioned in (2.2-e), with $v_{u}$ denoting the number of entries of $\widehat{u}$ being restricted, counting real and imaginary parts separately, and the maps $\widetilde{G}_{t}$ being the linear transformations containing the rows of DFT matrix in a specific arrangement. In our presentation, we generalize this class of constraint maps, $\widetilde{G}_{t}$, to be just smooth. For more detailed explanation see [PC17].

The frequency constraints on the state trajectories described in (2.2-d) are obtained in a similar manner. If one were to consider the DFT coefficients, one needs to use the DFT matrix corresponding to a signal of length $N+1$.

In the sequel we refer to the sequence $\left(u_{t}\right)_{t=0}^{N-1}$ as a control trajectory, with $u_{t}$ being the control action at time $t$.

\section{Preliminaries}

In this section we review some elementary facts from analysis in a nonsmooth setting; we refer the reader to [CLSW98, HUL93, Gül10] for further information on this topic.

For us $\langle\cdot, \cdot\rangle$ refers to the usual inner product in $\mathbb{R}^{n}$ and $\|\cdot\|$ to the norm induced by $\langle\cdot, \cdot\rangle$. For $\epsilon>0$ and $x \in \mathbb{R}^{n}, B_{\epsilon}(x)$ refers to the open ball $\left\{y \in \mathbb{R}^{n} \mid\|y-x\|<\epsilon\right\}$ of radius $\epsilon$ centered at $x$.

A function $g: \mathbb{R}^{n} \longrightarrow \mathbb{R}$ is said to be Lipschitz continuous near $x \in \mathbb{R}^{n}$ if there exists $r, L>0$ such that

$$
|g(y)-g(z)| \leqslant L\|y-z\| \quad \text { for all } y, z \in B_{r}(x) .
$$

A set $S \subset \mathbb{R}^{n}$ is convex if for all $x, y \in S$ and for all $\alpha \in[0,1]$, we have $(1-\alpha) x+\alpha y \in S$. A nonempty set $K \subset \mathbb{R}^{n}$ is a cone if for every $y \in K$ and for every $\alpha \geqslant 0$, we have $\alpha y \in K$. A set $K\left(x_{0}\right) \subset \mathbb{R}^{n}$ is a cone with vertex $x_{0} \in \mathbb{R}^{n}$ if for all $\alpha \geqslant 0$ and for all $y \in K\left(x_{0}\right)$, $\alpha\left(y-x_{0}\right) \in K\left(x_{0}\right)$. For a convex cone $K\left(x_{0}\right) \subset \mathbb{R}^{n}$ with vertex $x_{0}$, its dual cone $K\left(x_{0}\right)^{+}$is defined via polarity by

$$
K\left(x_{0}\right)^{+}:=\left\{y \in \mathbb{R}^{n} \mid\left\langle y, x-x_{0}\right\rangle \leqslant 0 \quad \text { for all } x \in K\left(x_{0}\right)\right\} .
$$

Definition 3.1. [Cla13, Section 1.4 on p. 20] Let $g: \mathbb{R}^{d} \times \mathbb{R}^{m} \longrightarrow \mathbb{R}^{n}$ be a continuous map and $g_{1}, \ldots, g_{n}$ are its components. For $y \in \mathbb{R}^{m}$ and a vector $v \in \mathbb{R}^{d}$, we denote by $\mathcal{D}_{v} g(\cdot, y)(x)$ the directional derivative of $g(\cdot, y)$ along $v$ at $x$, whenever the following limit exists:

$$
\begin{aligned}
\mathcal{D}_{v} g(\cdot, y)(x) & :=\lim _{\theta \downarrow 0} \frac{g(x+\theta v, y)-g(x, y)}{\theta} \\
& =\left(\lim _{\theta \downarrow 0} \frac{g_{1}(x+\theta v, y)-g_{1}(x, y)}{\theta}, \ldots, \lim _{\theta \downarrow 0} \frac{g_{n}(x+\theta v, y)-g_{n}(x, y)}{\theta}\right)^{\top}
\end{aligned}
$$

We note that the directional derivative above is defined as a right-hand (one-sided) limit. If $g$ is continuously differentiable, then $\mathcal{D}_{v} g(\cdot, y)(x)=\frac{\partial}{\partial x} g(x, y) \cdot v$. However, there are Lipschitz functions for which the left-hand and right-hand limit exist for all the directions at a point $x$ (which happens if the function is continuous at $x$ ), but they are not equal i.e., $\lim _{\theta \downarrow 0} \frac{g(x+\theta v)-g(x)}{\theta} \neq \lim _{\theta \uparrow 0} \frac{g(x+\theta v)-g(x)}{\theta}$. For example, for the absolute value function $\mathbb{R} \ni x \longmapsto$ $f(x)=|x| \in \mathbb{R}$ at $x=0, \mathcal{D}_{v} f(0)=|v|=\left\{\begin{array}{ll}v & \text { for } v \geqslant 0, \\ -v & \text { for } v<0,\end{array}\right.$ and $\lim _{\theta \uparrow 0} \frac{f(0+\theta v)-f(0)}{\theta}=-|v|$. Of course, $|\cdot|$ is not Gateaux differentiable at $x=0 .^{2}$

\footnotetext{
${ }^{2}$ Recall that if the right-hand limit of $g: \mathbb{R}^{d} \times \mathbb{R}^{m} \longrightarrow \mathbb{R}^{n}$ at $x$ is equal to the left-hand limit at $x$ then, the function $g$ is Gateaux differentiable at $x$. In this case, $\mathcal{D}_{v} g(\cdot, y)(x)=\frac{\partial}{\partial \xi} g(\cdot, y)(x) \cdot v$.
} 
Definition 3.2. If $h: \mathbb{R}^{n} \longrightarrow \mathbb{R}$ is a Lipschitz continuous function, then the generalized directional derivative $h^{\circ}(x ; v)$ of $h$ at $x$ along the direction $v$ is defined by

$$
h^{\circ}(x ; v):=\limsup _{\substack{y \rightarrow x \\ t \downarrow 0}} \frac{h(y+t v)-h(y)}{t} .
$$

In general, the generalized directional derivative takes values in $\mathbb{R} \cup\{+\infty\}$. The generalized gradient $\partial h(x)$ of $h$ at $x$ is a nonempty compact subset of $\left(\mathbb{R}^{n}\right)^{\star}$ defined by

$$
\partial h(x):=\left\{\xi \in\left(\mathbb{R}^{n}\right)^{\star} \mid\langle\xi, v\rangle \leqslant h^{\circ}(x ; v) \text { for all } v \in \mathbb{R}^{n}\right\} .
$$

Definition 3.3. A Lipschitz continuous function $g: \mathbb{R}^{d} \longrightarrow \mathbb{R}$ is said to be regular at $x \in \mathbb{R}^{d}$ if the directional derivative of $g$ at $x$ along any $v \in \mathbb{R}^{d}$ exists and is equal to its generalized directional derivative at $x$ along that $v$, i.e.,

$$
\mathcal{D}_{v} g(x)=g^{\circ}(x ; v)<+\infty .
$$

We look at two examples:

$\circ$ Let $\mathbb{R} \ni x \longmapsto f(x):=\max \{0, x\} \in \mathbb{R}$. It is clear that $f$ is Lipschitz continuous and is differentiable everywhere except at $x=0$. The directional derivative, the generalized directional derivative and the generalized gradient of $f$ at 0 and 1 are

$$
\begin{array}{ll}
\mathcal{D}_{v} f(0)=f(v), \quad f^{\circ}(0 ; v)=f(v), & \partial f(0)=[0,1], \\
\mathcal{D}_{v} f(1)=v, \quad f^{\circ}(1 ; v)=v \quad \text { and } \quad \partial f(1)=\{1\} .
\end{array}
$$

○ Let $\mathbb{R}^{d} \ni x \longmapsto f(x):=\|x\| \in \mathbb{R}$. Clearly, $f$ is a Lipschitz continuous function differentiable everywhere except at 0 . The generalized directional derivative and the generalized gradient of $f$ at 0 are

$$
\mathcal{D}_{v} f(0)=\|v\|, \quad f^{\circ}(0 ; v)=\|v\| \text { and } \quad \partial f(0)=\operatorname{cl}\left(B_{1}(0)\right) .
$$

Note that the functions in the above two examples are regular at 0.

Let $S \subset \mathbb{R}^{n}$ be a nonempty and closed set. The distance $d_{S}(x)$ of a point $x \in \mathbb{R}^{n}$ from $S$ is defined by

$$
\mathbb{R}^{n} \ni x \longmapsto d_{S}(x):=\inf _{s \in S}\|x-s\| \in[0,+\infty[.
$$

Definition 3.4. The Clarke tangent and normal cones to $S$ at a point $x \in S$, denoted by $T_{S}^{C}(x)$ and $N_{S}^{C}(x)$ respectively, are defined as

$$
\begin{aligned}
& T_{S}^{C}(x):=\left\{v \in \mathbb{R}^{n} \mid d_{S}^{\circ}(x ; v) \leqslant 0\right\}, \\
& N_{S}^{C}(x):=\left\{\xi \in\left(\mathbb{R}^{n}\right)^{\star} \mid\langle\xi, v\rangle \leqslant 0 \quad \text { for all } v \in T_{S}^{C}(x)\right\} .
\end{aligned}
$$

The Clarke tangent cone $T_{S}^{C}(x)$ at $x$ is a closed convex set and the normal cone $N_{S}^{C}(x)$ at $x$ is the polar of the tangent cone $T_{S}^{C}(x)$. Intuitively, the tangent cone at $x$ to $S$ is the set of directions along which it is possible to 'enter' $S$ from $x$, and the normal cone provides the set of directions along which one can most efficiently 'exit' $S$ from $x$. The Clarke tangent cone and normal cone to the sets $S_{1}=\left\{x \in \mathbb{R}^{2}\left|x_{2} \geqslant\right| x_{1} \mid\right\}$ and $S_{2}=\left\{x \in \mathbb{R}^{2}\left|x_{2} \leqslant 2\right| x_{1} \mid\right\}$ at origin $o=(0,0)$ are shown in Figure 1.

\section{Main Result}

Assumption 4.1. We stipulate that the maps $f_{t}$ 's in (2.1-c) are regular at every $(\xi, \mu) \in$ $\mathbb{R}^{d \times m}$. 

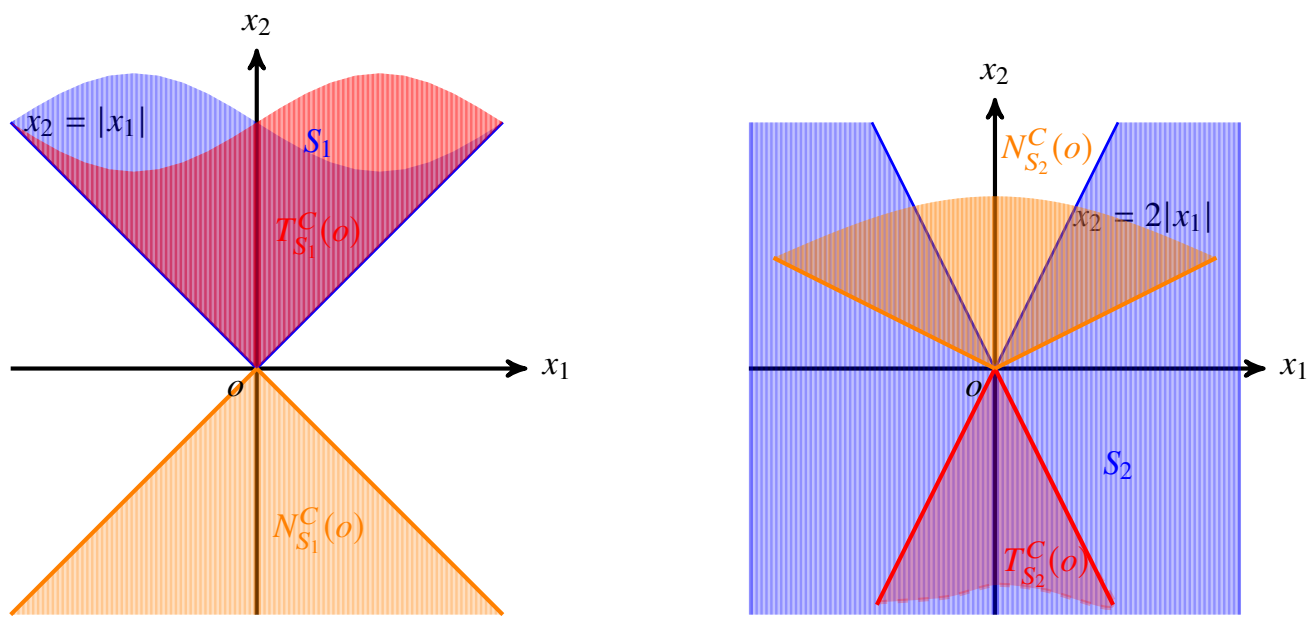

Figure 1. Tangent cone to the sets $S_{1}, S_{2}$ at $o=(0,0)$

Our main result is the following theorem: ${ }^{3}$

Theorem 4.2. Consider the problem (2.2) along with its associated data, and suppose that Assumption 4.1 holds. Let $\left(u_{t}^{*}\right)_{t=0}^{N-1}$ be a control trajectory that solves the optimal optimal control problem (2.2), and let $x^{*}:=\left(x_{t}^{*}\right)_{t=0}^{N}$ be the corresponding state trajectory. Define the Hamiltonian

(4.1)

$$
\begin{gathered}
\mathbb{R} \times\left(\mathbb{R}^{v_{x}}\right)^{\star} \times\left(\mathbb{R}^{v_{u}}\right)^{\star} \times\left(\mathbb{R}^{d}\right)^{\star} \times\{0, \ldots, N-1\} \times \mathbb{R}^{d} \times \mathbb{R}^{m} \ni\left(\alpha, \vartheta_{x}, \vartheta_{u}, \zeta, s, \xi, \mu\right) \longmapsto \\
H^{\alpha, \vartheta_{x}, \vartheta_{u}}(\zeta, s, \xi, \mu):=\left\langle\zeta, f_{s}(\xi, \mu)\right\rangle-\alpha c_{s}(\xi, \mu)-\left\langle\vartheta_{x}, \widetilde{F}_{s}(\xi)\right\rangle-\left\langle\vartheta_{u}, \widetilde{G}_{s}(\mu)\right\rangle \in \mathbb{R} .
\end{gathered}
$$

Then there exist

- an adjoint trajectory $\left(\eta_{t}^{f}\right)_{t=0}^{N-1} \subset\left(\mathbb{R}^{d}\right)^{\star}$,

- a sequence $\left(\eta_{t}^{x}\right)_{t=0}^{N} \subset\left(\mathbb{R}^{d}\right)^{\star}$, and

- a triplet $\left(\eta_{c}, \widehat{\eta^{x}}, \widehat{\eta^{u}}\right) \in \mathbb{R} \times\left(\mathbb{R}^{v_{x}}\right)^{\star} \times\left(\mathbb{R}^{v_{u}}\right)^{\star}$,

satisfying

(C-i) the non-negativity condition: $\eta_{c} \in\{0,1\}$;

(C-ii) the non-triviality condition: the adjoint trajectory $\left(\eta_{t}^{f}\right)_{t=0}^{N-1}$ and the triplet $\left(\eta_{c}, \widehat{\eta^{x}}, \widehat{\eta^{u}}\right)$ do not simultaneously vanish;

(C-iii) the state and adjoint dynamics:

$$
\begin{aligned}
& x_{t+1}^{*}=\frac{\partial}{\partial \zeta} H^{\eta_{c}, \widehat{\eta^{x}}, \widehat{\eta^{u}}}\left(\eta_{t}^{f}, t, x_{t}^{*}, u_{t}^{*}\right) \quad \text { for } t=0, \ldots, N-1, \\
& \left\langle\eta_{t-1}^{f}, y\right\rangle \geqslant \mathcal{D}_{y} H^{\eta_{c}, \widehat{\eta^{x}}, \widehat{\eta^{u}}}\left(\eta_{t}^{f}, t, \cdot, u_{t}^{*}\right)\left(x_{t}^{*}\right)-\left\langle\eta_{t}^{x}, y\right\rangle \\
& \quad \quad \text { for all } y \in \mathbb{R}^{d} \text { and some } \eta_{t}^{x} \in N_{\mathbb{S}_{t}}^{C}\left(x_{t}^{*}\right), \text { for } t=1, \ldots, N-1 ;
\end{aligned}
$$

${ }^{3}$ In the sequel there will arise the need to take partial derivatives of multivariable functions relative to specific variables, and we take care to precisely indicate the variables with respect to which we take these partial derivatives by introducing dummy variables in the definitions. The adjoint variables (a.k.a. 'multipliers') corresponding to the cost, the dynamics, the state-constraints, and the frequency constraints of the state and the control trajectories appear here, and we distinguish between them by introducing the different super-scripts of the single Greek letter $\eta$. Various objects in frequency space are distinguished by a 'hat'. While this mechanism leads to a multitude of sub-/super-scripts, we believe that it ensures much-needed transparency and tractability. 
(C-iv) the transversality conditions:

$$
\begin{gathered}
\mathcal{D}_{y} H^{\eta_{c}, \widehat{\eta^{x}}, \widehat{\eta^{u}}}\left(\eta_{0}^{f}, 0, \cdot, u_{0}^{*}\right)\left(x_{0}^{*}\right)-\left\langle\eta_{0}^{x}, y\right\rangle \leqslant 0 \\
\text { for all } y \in \mathbb{R}^{d} \text { and some } \eta_{0}^{x} \in N_{\mathbb{S}_{0}}^{C}\left(x_{0}^{*}\right), \text { and } \\
\eta_{N-1}^{f}=-\eta_{c} \frac{\partial}{\partial \xi} c_{N}\left(x_{N}^{*}\right)-\left(\frac{\partial}{\partial \xi} \widetilde{F}_{N}\left(x^{*}\right)\right)^{\top} \widehat{\eta^{x}}-\eta_{N}^{x} \\
\text { for some } \eta_{N}^{x} \in N_{\mathbb{S}_{N}}^{C}\left(x_{N}^{*}\right) ;
\end{gathered}
$$

(C-v) the Hamiltonian maximization condition:

$$
\mathcal{D}_{p} H^{\eta_{c}, \widehat{\eta^{x}}, \widehat{\eta^{u}}}\left(\eta_{t}^{f}, t, x_{t}^{*}, \cdot\right)\left(u_{t}^{*}\right) \leqslant 0 \quad \text { for all } p \in T_{\mathbb{U}_{t}}^{C}\left(u_{t}^{*}\right) \text { and for } t=0 \ldots, N-1 ;
$$

(C-vi) frequency constraints on the state trajectory $\left(x_{t}^{*}\right)_{t=0}^{N}$ :

$$
F\left(x_{0}^{*}, \ldots, x_{N}^{*}\right)=0 ;
$$

(C-vii) frequency constraints on the control action trajectory $\left(u_{t}^{*}\right)_{t=0}^{N-1}$ :

$$
G\left(u_{0}^{*}, \ldots, u_{N-1}^{*}\right)=0 .
$$

A detailed proof of Theorem 4.2 is postponed to the next section. In the remainder of the current section we shall briefly discuss the conditions $(\mathrm{C}-\mathrm{i})-(\mathrm{C}-\mathrm{vii})$ in the forthcoming remarks and examine several special cases of the main problem (2.2).

Remark 4.3. The scalar $\eta_{c}$, called the abnormal multiplier, takes the value 0 or 1 . If $\eta_{c}=0$, then the extremal lift $\left(\eta_{c},\left(\eta_{t}^{f}\right)_{t=0}^{N}, \widehat{\eta^{x}}, \widehat{\eta^{u}},\left(x_{t}^{*}\right)_{t=0}^{N},\left(u_{t}^{*}\right)_{t=0}^{N-1}\right)$ corresponding to an optimal pair $\left(\left(x_{t}^{*}\right)_{t=0}^{N},\left(u_{t}^{*}\right)_{t=0}^{N-1}\right)$ is called an abnormal extremal. If $\eta_{c}=1$, then the corresponding the extremal lift is called normal.

Remark 4.4. The entries of the sequence $\left(\eta_{t}^{f}\right)_{t=0}^{N-1}$ are called adjoint vectors or co-states; their evolution is governed by the adjoint dynamics (C-iii), and the transversality conditions (C-iv) provide its boundary conditions. Due to the nonsmooth nature of the state dynamics, the adjoint recursion is an inclusion

$$
\eta_{t-1}^{f} \in\left\{\eta \in \mathbb{R}^{d} \mid\langle\eta, y\rangle \geqslant \mathcal{D}_{y} H^{\eta_{c}, \widehat{\eta^{x}}, \widehat{\eta^{u}}}\left(\eta_{t}^{f}, t, \cdot, u_{t}^{*}\right)\left(x_{t}^{*}\right)-\left\langle\eta_{t}^{x}, y\right\rangle \text { for all } y \in \mathbb{R}^{d}\right\}
$$

as opposed to an equation. In Corollary 4.9 we shall observe that if the dynamics are continuously differentiable, then this inclusion turns into an equation, which is the standard adjoint equation in the classical Pontryagin maximum principle.

Remark 4.5. In the transversality condition (C-iv) the terms $N_{\mathbb{S}_{0}}^{C}\left(x_{0}^{*}\right)$ and $N_{\mathbb{S}_{N}}^{C}\left(x_{N}^{*}\right)$ are Clarke normal cones. However, there is a more general notion of a normal cone due to Mordukhovich called the basic or limiting normal cone [Mor06a, Chapter 1], and the approximate discrete-time PMP in [Mor06b] presents necessary conditions for optimality in terms of this limiting normal cone. Our approach of the proof uses the convexity of the Clarke normal cone in an essential way, while it is known that the limiting normal cone may fail to be convex; consequently, the result and its proof provided here does not carry over in an elegant fashion while involving the limiting normal cone.

Remark 4.6. The sequence of multipliers $\left(\eta_{t}^{x}\right)_{t=0}^{N}$ correspond to the pointwise state constraints. The definition (4.1) of the Hamiltonian features two new terms, $\left\langle\vartheta_{x}, \widetilde{F}_{s} \xi\right\rangle$ and $\left\langle\vartheta_{u}, \widetilde{G}_{s} \mu\right\rangle$, compared to the standard definition (e.g., [Bol75]). These terms account for the frequency constraints on the state trajectory and the control trajectory, respectively. One similar term also appeared in the Hamiltonian in [PC19], where frequency constraints on the control trajectories were considered. The vectors $\widehat{\eta^{x}}, \widehat{\eta^{u}}$ are the multipliers corresponding to the frequency constraints on the states and the controls, respectively. 
Remark 4.7. The condition (C-v) states that at every $t$ there exist a neighborhood of $u_{t}^{*}$ (say $\left.B_{\epsilon}\left(u_{t}^{*}\right)\right)$ such that

$$
H^{\eta_{c}, \widehat{\eta^{x}}, \widehat{\eta^{u}}}\left(\eta_{t}^{f}, t, x_{t}^{*}, u_{t}^{*}+p\right) \leqslant H^{\eta_{c}, \widehat{\eta^{x}}, \widehat{\eta^{u}}}\left(\eta_{t}^{f}, t, x_{t}^{*}, u_{t}^{*}\right) \quad \text { for all } p \in B_{\epsilon}\left(u_{t}^{*}\right) \cap T_{\mathbb{U}_{t}}^{C}\left(u_{t}^{*}\right) .
$$

The Clarke tangent cone $T_{\mathbb{U}_{t}}^{C}\left(u_{t}^{*}\right)$ provides a convex conic approximation of the set $\mathbb{U}_{t}$ at the point $u_{t}^{*}$. (Here the term approximation of a set $\mathbb{U}_{t}$ at a point $u_{t}^{*}$ stands for a set of directions along which it is possible to enter the set $\mathbb{U}_{t}$ from the point $u_{t}^{*}$.) Then the condition $(\mathrm{C}-\mathrm{v})$ implies that the value Hamiltonian at $u_{t}^{*}$ is greater than the value Hamiltonian at the points in the convex conic approximation of the set $\mathbb{U}_{t}$ at the point $u_{t}^{*}$ and that are close to $u_{t}^{*}$. Consequently, $(\mathrm{C}-\mathrm{v})$ does not imply Hamiltonian maximization which is a well-known phenomenon in the discrete time optimal control literature [MS04, Pše71]. Although not entirely appropriate, we call this condition "the Hamiltonian maximization" to maintain a similarity with the continuous time PMP. Besides, under suitable further assumptions on the admissible control action set, $(\mathrm{C}-\mathrm{v})$ implies maximization of the Hamiltonian. Indeed, if the admissible set $\mathbb{U}_{t}$ is convex and compact, then $\mathbb{U}_{t} \subset T_{\mathbb{U}_{t}}^{C}\left(u_{t}^{*}\right)$, and the condition $(\mathrm{C}-\mathrm{v})$ simplifies to

at every $t$ there exist a neighborhood of $u_{t}^{*}$, i.e., $B_{\epsilon}\left(u_{t}^{*}\right)$, such that

$$
H^{\eta_{c}, \widehat{\eta^{x}}, \widehat{\eta^{u}}}\left(\eta_{t}^{f}, t, x_{t}^{*}, u_{t}^{*}+p\right) \leqslant H^{\eta_{c}, \widehat{\eta^{x}}, \widehat{\eta^{u}}}\left(\eta_{t}^{f}, t, x_{t}^{*}, u_{t}^{*}\right) \quad \text { for all } p \in B_{\epsilon}\left(u_{t}^{*}\right) \cap \mathbb{U}_{t} \text {. }
$$

In other words, $u_{t}^{*}$ is a local maximizer of the Hamiltonian at time $t$. Therefore, if we assume that at each $t$ the Hamiltonian 4.1 is concave with respect to the control variable then the condition $(\mathrm{C}-\mathrm{v})$ implies the local maximization of the Hamiltonian at each instant along the optimal trajectories.

Corollary 4.8. Suppose that $\left(\eta_{c},\left(\eta_{t}^{f}\right)_{t=0}^{N}, \widehat{\eta^{x}}, \widehat{\eta^{u}},\left(x_{t}^{*}\right)_{t=0}^{N},\left(u_{t}^{*}\right)_{t=0}^{N-1}\right)$ is an extremal of (2.2) and let $H^{\eta_{c}}, \widehat{\eta^{x}}, \widehat{\eta^{u}}$ be the Hamiltonian defined in (4.1).

(i) If the dynamics $f_{i}$ in (2.1-c) for every $i \in\{1, \ldots, N-1\}$ are smooth with respect to the state variable $\xi$ at $x_{i}^{*}$, then the adjoint dynamics in (C-iii) of Theorem 4.2 at $t=i$ strengthens to the recursion

$$
\eta_{i-1}^{f}=\frac{\partial}{\partial \xi} H^{\eta_{c}, \widehat{\eta^{x}}, \widehat{\eta^{u}}}\left(\eta_{i}^{f}, i, x_{i}^{*}, u_{i}^{*}\right)-\eta_{i}^{x} \quad \text { for some } \eta_{i}^{x} \in N_{\mathbb{S}_{i}}^{C}\left(x_{i}^{*}\right) .
$$

(ii) If the dynamics $f_{0}$ in $(2.1-\mathrm{c})$ is smooth with respect to the state variable $\xi$ at $x_{0}^{*}$, then the transversality condition $(\mathrm{C}-\mathrm{iv})$ of Theorem 4.2 strengthens to:

$$
\begin{aligned}
& \frac{\partial}{\partial \xi} H^{\eta_{c}, \widehat{\eta^{x}}, \widehat{\eta^{u}}}\left(\eta_{0}^{f}, 0, x_{0}^{*}, u_{0}^{*}\right)=\eta_{0}^{x} \quad \text { for some } \eta_{0}^{x} \in N_{\mathbb{S}_{0}}^{C}\left(x_{0}^{*}\right), \\
& \eta_{N-1}^{f}=-\eta_{c} \frac{\partial}{\partial \xi} c_{N}\left(x_{N}^{*}\right)-\left(\frac{\partial}{\partial \xi} \widetilde{F}_{N}\left(x^{*}\right)\right)^{\top} \widehat{\eta^{x}}-\eta_{N}^{x} \quad \text { for some } \eta_{N}^{x} \in N_{\mathbb{S}_{N}}^{C}\left(x_{N}^{*}\right) .
\end{aligned}
$$

(iii) If the dynamics $f_{j}$ in (2.1-c) for every $j \in\{0, \ldots, N-1\}$ are smooth with respect to the control variable $\mu$ at $u_{j}^{*}$, then the Hamiltonian maximization $(\mathrm{C}-\mathrm{v})$ condition in Theorem 4.2 at $t=j$ simplifies to:

$$
\left\langle\frac{\partial}{\partial \mu} H^{\eta_{c}, \widehat{\eta^{x}}, \widehat{\eta^{u}}}\left(\eta_{j}^{f}, j, x_{j}^{*}, u_{j}^{*}\right), p\right\rangle \leqslant 0 \quad \text { for all } p \in T_{\mathbb{U}_{j}}^{C}\left(u_{j}^{*}\right) .
$$

Proof. (i) For $i \in\{1, \ldots, N-1\}$ if the dynamics (2.1-c) is smooth with respect to $\xi$ at $x_{i}^{*}$ then Hamiltonian $H^{\eta_{c}, \widehat{\eta^{x}}, \widehat{\eta^{u}}}$ in (4.1) is smooth with respect to $\xi$ at $x_{i}^{*}$. Since the Hamiltonian is smooth at $x_{i}^{*}$, the directional derivative of $H^{\eta_{c}, \widehat{\eta^{x}}, \widehat{\eta^{u}}}\left(\eta_{i}^{f}, i, \cdot, u_{i}\right)$ at $x_{i}^{*}$ in any direction $y \in \mathbb{R}^{d}$ is the inner product of the gradient of $H^{\eta_{c}}, \widehat{\eta^{x}}, \widehat{\eta^{u}}\left(\eta_{i}^{f}, i, \cdot, u_{i}\right)$ at 
$x_{i}^{*}$ and $y$. In other words, referring to the definition of the Hamiltonian $H^{\eta_{c}, \widehat{\eta^{x}}, \widehat{\eta^{u}}}$ in (4.1) and the directional derivative in (3.1),

$$
\mathcal{D}_{y} H^{\eta_{c}, \widehat{\eta^{x}}, \widehat{\eta^{u}}}\left(\eta_{i}^{f}, i, \cdot, u_{i}^{*}\right)\left(x_{i}^{*}\right)=\left\langle\frac{\partial}{\partial \xi} H^{\eta_{c}, \widehat{\eta^{x}}, \widehat{\eta^{u}}}\left(\eta_{i}^{f}, i, x_{i}^{*}, u_{i}^{*}\right), y\right\rangle \text {. }
$$

The condition (C-iii) of Theorem 4.2 on adjoint dynamics gives us

$$
\left\langle\eta_{i-1}^{f}-\frac{\partial}{\partial \xi} H^{\eta_{c}, \widehat{\eta^{x}}, \widehat{\eta^{u}}}\left(\eta_{i}^{f}, i, x_{i}^{*}, u_{i}^{*}\right)+\eta_{i}^{x}, y\right\rangle \geqslant 0 \quad \text { for all } y \in \mathbb{R}^{d} .
$$

Since the inner product is linear in $y$ and the preceding inequality is true for all $y \in \mathbb{R}^{d}$, we may replace $y$ with $-y$ in the above inequality, leading to

$$
\eta_{i-1}^{f}-\frac{\partial}{\partial \xi} H^{\eta_{c}, \widehat{\eta^{x}}, \widehat{\eta^{u}}}\left(\eta_{i}^{f}, i, x_{i}^{*}, u_{i}^{*}\right)+\eta_{i}^{x}=0
$$

as desired.

(ii) For $t=0$ if the dynamics (2.1-c) is smooth with respect to state variable $\xi$ at $x_{0}^{*}$ then the Hamiltonian $H^{\eta_{c}, \widehat{\eta^{x}}, \widehat{\eta^{u}}}$ in (4.1) is continuously differentiable with respect to $\xi$ at $x_{0}^{*}$. Therefore, the directional derivative of Hamiltonian $H^{\eta_{c}, \widehat{\eta^{x}}, \widehat{\eta^{u}}}\left(\eta_{0}^{f}, 0, \cdot, u_{0}\right)$ at $x_{0}^{*}$ in any direction $y \in \mathbb{R}^{d}$ is

$$
\mathcal{D}_{y} H^{\eta_{c}, \widehat{\eta^{x}}, \widehat{\eta^{u}}}\left(\eta_{0}^{f}, 0, \cdot, u_{0}^{*}\right)\left(x_{0}^{*}\right)=\left\langle\frac{\partial}{\partial \xi} H^{\eta_{c}, \widehat{\eta^{x}}, \widehat{\eta^{u}}}\left(\eta_{0}^{f}, 0, x_{0}^{*}, u_{0}^{*}\right), y\right\rangle .
$$

Then the transversality condition (C-iv) of Theorem 4.2 and the linearity of the above inner product with respect to $y$ leads to

$$
\frac{\partial}{\partial \xi} H^{\eta_{c}, \widehat{\eta^{x}}, \widehat{\eta^{u}}}\left(\eta_{0}^{f}, 0, x_{0}^{*}, u_{0}^{*}\right)=\eta_{0}^{x} .
$$

(iii) For $j \in\{0, \ldots, N-1\}$ if the dynamics $f_{j}$ in (2.1-c) is smooth with respect to the control variable $\mu$ at $u_{j}^{*}$, then we can write the directional derivative of the Hamiltonian

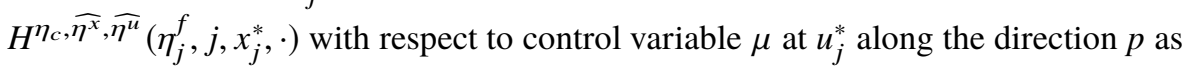
the inner product

$$
\mathcal{D}_{p} H^{\eta_{c}, \widehat{\eta^{x}}, \widehat{\eta^{u}}}\left(\eta_{j}^{f}, j, x_{j}^{*}, \cdot\right)\left(u_{j}^{*}\right)=\left\langle\frac{\partial}{\partial \mu} H^{\eta_{c}, \widehat{\eta^{x}}, \widehat{\eta^{u}}}\left(\eta_{j}^{f}, j, x_{j}^{*}, u_{j}^{*}\right), p\right\rangle .
$$

Then for $t=j$ the Hamiltonian maximization condition $(\mathrm{C}-\mathrm{v})$ of Theorem 4.2 specializes to

$$
\left\langle\frac{\partial}{\partial \mu} H^{\eta_{c}, \widehat{\eta^{x}}, \widehat{\eta^{u}}}\left(\eta_{j}^{f}, j, x_{j}^{*}, u_{j}^{*}\right), p\right\rangle \leqslant 0 .
$$

Remark 4.9. Suppose that in (2.2) we replace the Lipschitz continuous dynamics hypothesis (2.1-c) of (2.1) with each

$$
\mathbb{R}^{d} \times \mathbb{R}^{m} \ni(\xi, \mu) \longmapsto f_{t}(\xi, \mu) \in \mathbb{R}^{d} \quad \text { for } t=0, \ldots, N-1,
$$

being continuously differentiable, then the Hamiltonian as defined in (4.1) is continuously differentiable in $\xi$ and $\mu$. In this setting, the conditions (C-iii), (C-iv) and (C-v) of Theorem 4.2 can be strengthened to $\left(\mathrm{iii}^{*}\right),\left(\mathrm{iv}^{*}\right)$ and $\left(\mathrm{v}^{*}\right)$ respectively, given below:

(iii*) state and adjoint dynamics:

$$
\begin{aligned}
& x_{t+1}^{*}=\frac{\partial}{\partial \zeta} H^{\eta_{c}, \widehat{\eta^{x}}, \widehat{\eta^{u}}}\left(\eta_{t}^{f}, t, x_{t}^{*}, u_{t}^{*}\right) \quad \text { for } t=0, \ldots, N-1, \\
& \eta_{t-1}^{f}=\frac{\partial}{\partial \xi} H^{\eta_{c}, \widehat{\eta^{x}}, \widehat{\eta^{u}}}\left(\eta_{t}^{f}, t, x_{t}^{*}, u_{t}^{*}\right)-\eta_{t}^{x}
\end{aligned}
$$

for some $\eta_{t}^{x} \in N_{\mathbb{S}_{t}}^{C}\left(x_{t}^{*}\right)$ and for $t=1 \ldots, N-1$; 
$\left(\mathrm{iv}^{*}\right)$ transversality conditions:

$$
\begin{aligned}
& \frac{\partial}{\partial \xi} H^{\eta_{c}, \widehat{\eta^{x}}, \widehat{\eta^{u}}}\left(\eta_{0}^{f}, 0, x_{0}^{*}, u_{0}^{*}\right)=\eta_{0}^{x} \quad \text { for all } y \in \mathbb{R}^{d} \text { and for some } \eta_{0}^{x} \in N_{\mathbb{S}_{0}}^{C}\left(x_{0}^{*}\right), \\
& \eta_{N-1}^{f}=-\eta_{c} \frac{\partial}{\partial \xi} c_{N}\left(x_{N}^{*}\right)-\left(\frac{\partial}{\partial \xi} \widetilde{F}_{N}\left(x^{*}\right)\right)^{\top} \widehat{\eta^{x}}-\eta_{N}^{x} \quad \text { for some } \eta_{N}^{x} \in N_{\mathbb{S}_{N}}^{C}\left(x_{N}^{*}\right) ;
\end{aligned}
$$

$\left(\mathrm{v}^{*}\right)$ Hamiltonian maximization condition:

$$
\left\langle\frac{\partial}{\partial \mu} H^{\eta_{c}, \widehat{\eta^{x}}, \widehat{\eta^{u}}}\left(\eta_{t}^{f}, t, x_{t}^{*}, u_{t}^{*}\right), p\right\rangle \leqslant 0 \quad \text { for all } p \in T_{\cup_{t}}^{C}\left(u_{t}^{*}\right) \text { and for } t=0 \ldots, N-1 .
$$

We have a second (immediate) special case, whose proof follows at once from the preceding discussion.

Corollary 4.10. If the controlled system in (2.2) is linear, that is, (2.1) is replaced by

$$
x_{t+1}=A_{t} x_{t}+B_{t} u_{t} \quad \text { where } A_{t} \in \mathbb{R}^{d \times d} \text { and } \quad B_{t} \in \mathbb{R}^{d \times m} \text { for } t=0, \ldots, N-1 \text {, }
$$

then for the Hamiltonian defined in (4.1), the assertions of Theorem 4.2 hold with the adjoint dynamics in (C-iii) given by

$$
\eta_{t-1}^{f}=A_{t}^{\top} \eta_{t}^{f}-\eta_{c} \frac{\partial}{\partial \xi_{t}} c_{t}\left(x_{t}^{*}, u_{t}^{*}\right)-\widetilde{F}_{t}\left(x_{t}^{*}\right)^{\top} \widehat{\eta^{x}}-\eta_{t}^{x} \quad \text { for } t=0, \ldots, N-1 .
$$

Moreover, if each $\mathbb{U}_{t}$ is non-empty, convex, and compact, then the condition $(\mathrm{C}-\mathrm{v})$ in Theorem 4.2 becomes the standard Hamiltonian maximization condition

$$
H^{\eta_{c}, \widehat{\eta^{x}}, \widehat{\eta^{u}}}\left(\eta_{t}^{f}, t, x_{t}^{*}, u_{t}^{*}\right)=\max _{\mu \in \mathbb{U}_{t}} H^{\eta_{c}, \widehat{\eta^{x}}, \widehat{\eta^{u}}}\left(\eta_{t}^{f}, t, x_{t}^{*}, \mu\right) \quad \text { for } t=0, \ldots, N-1 .
$$

The following result addresses the optimal control problem (2.2) under a different set of hypotheses than Assumption 4.1. Here we assume that

- the dynamics are smooth and

- the cost functions are regular but may be nonsmooth

while retaining the rest of the problem data. We observe that the necessary conditions for this modified problem are similar to the necessary conditions in Theorem 4.2 except the transversality conditions; these necessary condition cater to, e.g., $\ell_{1}$-minimization problems that may be employed to enforce sparsity. The precise statement is as follows:

Theorem 4.11. Consider the problem (2.2) with the following modifications:

(a) the functions $f_{t}$ in (2.1) are continuously differentiable everywhere, and

(b) the functions $c_{t}$ in (2.2-a) are regular at every $(\xi, \mu) \in \mathbb{R}^{d \times m} \cdot{ }^{4}$

Let $\left(u_{t}^{*}\right)_{t=0}^{N-1}$ be a control trajectory that solves the optimal optimal control problem (2.2) with the modifications (a) and (b), and let $x^{*}:=\left(x_{t}^{*}\right)_{t=0}^{N}$ be the corresponding state trajectory. Then, for the Hamiltonian defined in (4.1), there exist

- an adjoint trajectory $\left(\eta_{t}^{f}\right)_{t=0}^{N-1} \subset\left(\mathbb{R}^{d}\right)^{\star}$,

- a sequence $\left(\eta_{t}^{x}\right)_{t=0}^{N} \subset\left(\mathbb{R}^{d}\right)^{\star}$, and

- a triplet $\left(\eta_{c}, \widehat{\eta^{x}}, \widehat{\eta^{u}}\right) \in \mathbb{R} \times\left(\mathbb{R}^{v_{x}}\right)^{\star} \times\left(\mathbb{R}^{v_{u}}\right)^{\star}$,

satisfying

(i) the non-negativity condition $\eta_{c} \in\{0,1\}$;

(ii) the non-triviality condition the adjoint trajectory $\left(\eta_{t}^{f}\right)_{t=0}^{N-1}$ and the triplet $\left(\eta_{c}, \widehat{\eta^{x}}, \widehat{\eta^{u}}\right)$ do not simultaneously vanish;

(iii) the state and adjoint dynamics (C-iii);

\footnotetext{
${ }^{4} \mathrm{We}$ emphasize that the assumption of continuous differentiability of the cost functions is being removed; consequently, $c_{t}$ may fail to be differentiable at some $(\xi, \mu) \in \mathbb{R}^{d \times m}$.
} 
(iv) the transversality conditions

$$
\begin{gathered}
\mathcal{D}_{y} H^{\eta_{c}, \widehat{\eta^{x}}, \widehat{\eta^{u}}}\left(\eta_{0}^{f}, 0, \cdot, u_{0}^{*}\right)\left(x_{0}^{*}\right)-\left\langle\eta_{0}^{x}, y\right\rangle \leqslant 0 \\
\quad \text { for all } y \in \mathbb{R}^{d} \text { and some } \eta_{0}^{x} \in N_{\mathbb{S}_{0}}^{C}\left(x_{0}^{*}\right), \text { and } \\
\left\langle\eta_{N-1}^{f}, y\right\rangle+\eta_{c} \mathcal{D}_{y} c_{N}\left(x_{N}^{*}\right)+\left\langle\left(\frac{\partial}{\partial \xi} \widetilde{F}_{N}\left(x^{*}\right)\right)^{\top} \widehat{\eta^{x}}+\eta_{N}^{x}, y\right\rangle \geqslant 0 \\
\text { for all } y \in \mathbb{R}^{d} \text { and some } \eta_{N}^{x} \in N_{\mathbb{S}_{N}}^{C}\left(x_{N}^{*}\right) ;
\end{gathered}
$$

(v) the Hamiltonian maximization condition $(\mathrm{C}-\mathrm{v})$;

(vi) frequency constraints on the state trajectory $\left(x_{t}^{*}\right)_{t=0}^{N}(\mathrm{C}-\mathrm{vi})$;

(vii) frequency constraints on the control action trajectory $\left(u_{t}^{*}\right)_{t=0}^{N-1}(\mathrm{C}$-vii).

Proof. A proof of the above theorem follows the steps of the proof of the Theorem 4.2 given in the next section; we omit the details in the interest of brevity. The proof will follow the steps similar to the proof of Theorem 4.2 present in the next section. To summarize, we start with Step I in §5.1, and follow Step II in §5.2 until the Claim. In the Claim itself, the nonsmooth cost and smooth dynamics are changed from (5.12) into the following condition:

$$
0 \in \eta_{c} \partial C\left(z^{*}\right)+\frac{\partial}{\partial z}\left\langle\lambda^{f}, f_{d}(\cdot)\right\rangle\left(z^{*}\right)+\left(\frac{\partial}{\partial z} \widehat{F}\left(z^{*}\right)\right)^{\top} \widehat{\eta^{x}}+\left(\frac{\partial}{\partial z} \widehat{G}\left(z^{*}\right)\right)^{\top} \widehat{\eta^{u}}+N_{\Omega}^{C}\left(z^{*}\right) .
$$

For the above new condition, we define the function $h(z):=\sum_{t=0}^{N-1} h_{t}(z)=\sum_{t=0}^{N-1} \eta_{c} c_{t}\left(x_{t}, u_{t}\right)$ and continue Step II, followed by Step III in $\$ 5.3$ to arrive at the end result.

\section{Proof of Theorem 4.2}

In this section we provide a detailed proof of the main result Theorem 4.2. Flowchart 2 gives an idea of a proof, and we elaborate the main steps below:

Sketch of the proof: We proceed as per the three steps below:

Step (I) Our optimal control problem is lifted to an equivalent optimization problem in a suitable high-dimensional product space.

Step (II) First order necessary conditions for the optimization problem in Step (I) are obtained using Clarke's necessary condition for non-smooth optimization problems.

Step (III) The necessary conditions obtained in Step (II) are projected to appropriate factors to arrive at necessary condition of the original control problem.

5.1. Step (I). Equivalent optimization problem: The objective of this step is to transform the original optimal control problem (2.2) to an equivalent optimization problem. The approach is to lift the optimal control problem to an appropriate high-dimensional product space. Here by "lift" we mean the concatenation of $N+1$ vectors (say $\xi_{0}, \ldots, \xi_{N}$ ) from the space $\mathbb{R}^{d}$ corresponding to the states and $N$ vectors (say $\mu_{0}, \ldots, \mu_{N-1}$ ) from the space $\mathbb{R}^{m}$ corresponding to the control actions. Thus, every vector in the lifted high-dimensional space $\mathbb{R}^{v}$ is of the form

$$
z:=\left(\xi_{0}, \ldots, \xi_{N}, \mu_{0}, \ldots, \mu_{N-1}\right) \in \mathbb{R}^{v},
$$

where $v:=d(N+1)+m N,\left(\xi_{t}\right)_{t=0}^{N} \subset \mathbb{R}^{d}$ and $\left(\mu_{t}\right)_{t=0}^{N-1} \subset \mathbb{R}^{m}$. For brevity of notation, we define $z:=(\bar{\xi}, \bar{\mu})$, where $\bar{\xi}:=\left(\xi_{0}, \ldots, \xi_{N}\right) \in \mathbb{R}^{d(N+1)}$ and $\bar{\mu}:=\left(\mu_{0}, \ldots, \mu_{N-1}\right) \in \mathbb{R}^{m N}$.

In order to extract a vector $\xi_{t} \in \mathbb{R}^{d}$ for some $t \in\{0, \ldots, N\}$ and $\mu_{t} \in \mathbb{R}^{m}$ for some $t \in\{0, \ldots, N-1\}$ from $z$, we employ standard state projection maps and control projection 


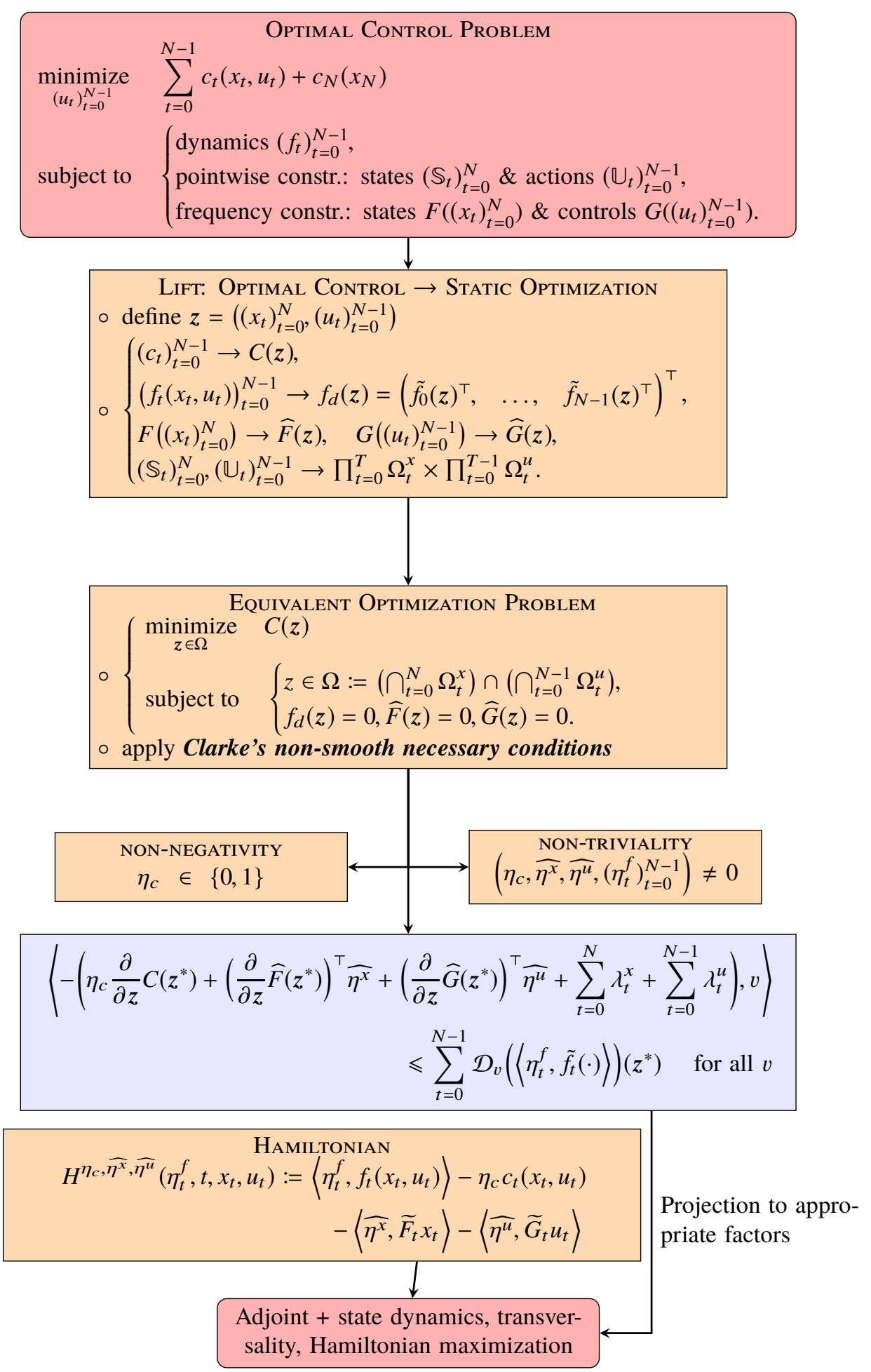

Figure 2. Flowchart of a proof 
maps defined by

$$
\begin{aligned}
& \mathbb{R}^{v} \ni \boldsymbol{z}=(\bar{\xi}, \bar{\mu}) \longmapsto \pi_{t}^{x}(\boldsymbol{z}):=\xi_{t} \in \mathbb{R}^{d} \quad \text { for } t=0, \ldots, N, \\
& \mathbb{R}^{v} \ni \boldsymbol{z}=(\bar{\xi}, \bar{\mu}) \longmapsto \pi_{t}^{u}(\boldsymbol{z}):=\mu_{t} \in \mathbb{R}^{m} \quad \text { for } t=0, \ldots, N-1,
\end{aligned}
$$

In the above notation the superscript $x$ or $u$ of the projection map $\pi$ indicates the space of states $x$ or control action $u$ respectively, and the subscript denotes the time instance.

Let us define the functions and the sets involved in the optimal control problem in the space $\mathbb{R}^{v}$ to arrive at an equivalent optimization problem.

- The lift of the total cost: Define the function

$$
\mathbb{R}^{v} \ni z \longmapsto C(z):=C(\bar{\xi}, \bar{\mu})=\sum_{t=0}^{N-1} c_{t}\left(\xi_{t}, \mu_{t}\right)+c_{N}\left(\xi_{T}\right) \in \mathbb{R} .
$$

- The lift of the dynamics: Define the function

$$
\mathbb{R}^{v} \ni z \longmapsto f_{d}(z):=\left(\tilde{f}_{0}(z)^{\top}, \quad \ldots, \quad \tilde{f}_{N-1}(z)^{\top}\right)^{\top} \in \mathbb{R}^{d N},
$$

where

$$
\mathbb{R}^{v} \ni \boldsymbol{z} \longmapsto \tilde{f}_{t}(z):=\xi_{t+1}-f_{t}\left(\xi_{t}, \mu_{t}\right) \in \mathbb{R}^{d} \text { for each } t=0, \ldots N-1 .
$$

Clearly, if a vector $z=\left(\xi_{0}, \ldots, \xi_{N}, \mu_{0}, \ldots, \mu_{N-1}\right)$ belongs to the set given by $\{y \in$ $\left.\mathbb{R}^{v} \mid f_{d}(y)=0\right\}$, with the state and control projections $\left(\xi_{t}\right)_{t=0}^{N},\left(\mu_{t}\right)_{t=0}^{N-1}$ then $\left(\xi_{t}\right)_{t=1}^{N}$ is a solution of the dynamical system (2.1), corresponding to the initial condition $\xi_{0}$ and the control sequence $\left(\mu_{t}\right)_{t=0}^{N-1}$. Similarly, if $\left(\mu_{t}\right)_{t=0}^{N-1}$ is an admissible control sequence and with $\left(\xi_{t}\right)_{t=0}^{N-1}$ being the corresponding solution of the dynamical system (2.1), for the initial condition $\xi_{0}$ then the concatenated vector $z:=\left(\xi_{0}, \ldots, \xi_{N}, \mu_{0}, \ldots, \mu_{N-1}\right)$ belongs to the set $\left\{y \in \mathbb{R}^{v} \mid f_{d}(y)=0\right\}$. Therefore, the dynamics (2.1) in the optimal control problem (2.2) can be equivalently modeled by an equality constraint $f_{d}(\boldsymbol{z})=0$ in $\mathbb{R}^{v}$.

- The lift of the sets corresponding to point-wise state and control action constraints: Define the sets

$$
\begin{array}{ll}
\Omega_{t}^{x}:=\left\{z \in \mathbb{R}^{v} \mid \pi_{t}^{x}(z) \in \mathbb{S}_{t}\right\} & \text { for } t=0, \ldots, N, \\
\Omega_{t}^{u}:=\left\{z \in \mathbb{R}^{v} \mid \pi_{t}^{x}(z) \in \mathbb{U}_{t}\right\} & \text { for } t=0, \ldots, N-1 .
\end{array}
$$

Observe that if the sets $\left(\mathbb{S}_{t}\right)_{t=0}^{N}$ and $\left(\mathbb{U}_{t}\right)_{t=0}^{N-1}$ are closed then the corresponding lifted sets $\left(\Omega_{t}^{x}\right)_{t=0}^{N}$ and $\left(\Omega_{t}^{u}\right)_{t=0}^{N-1}$ are also closed. Hence the closedness of sets is preserved under the defined lift.

Further any vector $z=\left(\xi_{0}, \ldots, \xi_{N}, \mu_{0}, \ldots, \mu_{N-1}\right) \in \Omega_{t}^{x}$ for some $t \in\{0, \ldots, N\}$ if and only if the corresponding state projection $\xi_{t} \in \mathbb{R}^{d}$ satisfies the state constraints given by (2.2-b) (i.e., $\left.\xi_{t} \in \mathbb{S}_{t}\right)$. Similarly for any $z=\left(\xi_{0}, \ldots, \xi_{N}, \mu_{0}, \ldots, \mu_{N-1}\right) \in \Omega_{t}^{u}$ for some $t \in\{0, \ldots, N-1\}$ if and only if the corresponding control projection $\mu_{t} \in \mathbb{R}^{m}$ satisfies the control constraints given by (2.2-c) (i.e., $\mu_{t} \in \mathbb{U}_{t}$ ).

Therefore $z=\left(\xi_{0}, \ldots, \xi_{N}, \mu_{0}, \ldots, \mu_{N-1}\right) \in\left(\cap_{t=0}^{N} \Omega_{t}^{x}\right) \cap\left(\cap_{t=0}^{N-1} \Omega_{t}^{u}\right)$ if and only if $\xi_{t} \in \mathbb{S}_{t}$ for $t=0, \ldots, N$, and $\mu_{t} \in \mathbb{U}_{t}$ for $t=0, \ldots, N-1$. Hence the point-wise state and control constraints is equivalently given by the constraint $z \in\left(\cap_{t=0}^{N} \Omega_{t}^{x}\right) \cap\left(\cap_{t=0}^{N-1} \Omega_{t}^{u}\right)$ in $\mathbb{R}^{v}$.

- The lift of the frequency constraints on state and control trajectory: Define the functions

$$
\begin{aligned}
& \mathbb{R}^{v} \ni z \longmapsto \widehat{F}(z):=F\left(\pi_{0}^{x}(z), \ldots, \pi_{N}^{x}(z)\right) \in \mathbb{R}^{v_{x}} \text { and } \\
& \mathbb{R}^{v} \ni z \longmapsto \widehat{G}(z):=G\left(\pi_{0}^{u}(z), \ldots, \pi_{N-1}^{u}(z)\right) \in \mathbb{R}^{v_{u}} .
\end{aligned}
$$

From the definition of $\widehat{F}$ and $\widehat{G}$ it is clear that the equality constraints $\widehat{F}(z)=0$ and $\widehat{G}(z)=0$ in $\mathbb{R}^{v}$ are equivalent to the state frequency constraints (2.2-d) and the control frequency constraints (2.2-e), respectively. 
In view of the various definitions above, we define the optimization problem

$\begin{array}{|ll|}\underset{z \in \Omega}{\operatorname{minimize}} & C(z) \\ \text { subject to } & \left\{\begin{array}{l}\Omega:=\left(\bigcap_{t=0}^{N} \Omega_{t}^{x}\right) \cap\left(\bigcap_{t=0}^{N-1} \Omega_{t}^{u}\right), \\ f_{d}(z)=0, \\ \widehat{F}(z)=0, \\ \widehat{G}(z)=0,\end{array}\right.\end{array}$

Note that the cost and the constraints of (5.9) and (2.2) are identical; consequently, these are equivalent problems.

\subsection{Step (II). Necessary condition for the equivalent optimization problem:}

Let $z^{*}$ denote a solution to the optimization problem (5.9), comprising of an optimal control sequence $\left(u_{t}^{*}\right)_{t=0}^{N-1}$ and its corresponding state trajectory $\left(x_{t}^{*}\right)_{t=0}^{N}$; that is, $z^{*}=$ $\left(x_{0}^{*}, \cdots, x_{N}^{*}, u_{0}^{*}, \cdots, u_{N-1}^{*}\right)$. Since (5.9) is equivalent to the optimal control problem (2.2), if $z^{*}$ is a solution to the optimization problem (5.9), then $\left(u_{t}^{*}\right)_{t=0}^{N-1}$ is a solution to the optimal control problem (2.2) and $\left(x_{t}^{*}\right)_{t=0}^{N}$ is its corresponding optimal state trajectory.

The following theorem ( $\S \mathrm{A} .1)$ ) provides necessary condition for $z^{*}$ to be a solution of

Theorem 5.1. If $z^{*}$ is a solution to the optimization problem (5.9), then there exists a non-trivial vector $\left(\eta_{c}, \lambda^{f}, \widehat{\eta^{x}}, \widehat{\eta^{u}}\right) \in\{0,1\} \times\left(\mathbb{R}^{d N}\right)^{\star} \times\left(\mathbb{R}^{v_{x}}\right)^{\star} \times\left(\mathbb{R}^{v_{u}}\right)^{\star}$ such that

$$
0 \in \partial\left(\eta_{c} C(\cdot)+\left\langle\lambda^{f}, f_{d}(\cdot)\right\rangle+\left\langle\widehat{\eta^{x}}, \widehat{F}(\cdot)\right\rangle+\left\langle\widehat{\eta^{u}}, \widehat{G}(\cdot)\right\rangle\right)\left(z^{*}\right)+N_{\Omega}^{C}\left(z^{*}\right) .
$$

In the setting of Theorem 5.1, the various scalars/vectors, $\eta_{c}, \lambda^{f}, \widehat{\eta^{x}}, \widehat{\eta^{u}}$ are called multipliers corresponding to the lifted $\operatorname{cost} C$, the function $f_{d}$, the lifted state frequency constraints $\widehat{F}$, and the lifted control frequency constraints $\widehat{G}$, respectively.

The condition (5.10) is a set theoretic necessary condition for optimality, which means that there exist some non-trivial vectors in the sets

$$
\partial\left(\eta_{c} C(\cdot)+\left\langle\lambda^{f}, f_{d}(\cdot)\right\rangle+\left\langle\widehat{\eta^{x}}, \widehat{F}(\cdot)\right\rangle+\left\langle\widehat{\eta^{u}}, \widehat{G}(\cdot)\right\rangle\right)\left(z^{*}\right) \text { and } N_{\Omega}^{C}\left(z^{*}\right)
$$

such that their sum is zero. In order to simplify the condition above we characterize the elements of these sets.

○ Consider, first, the set $N_{\Omega}^{C}\left(z^{*}\right)$. We have the following characterization of $N_{\Omega}^{C}\left(z^{*}\right)$ :

Claim: For any vector $\gamma \in N_{\Omega}^{C}\left(z^{*}\right)$, there exist vectors $\gamma_{t}^{x} \in N_{\Omega_{t}^{x}}^{C}\left(z^{*}\right)$ and $\gamma_{t}^{u} \in$ $N_{\Omega_{t}^{u}}^{C}\left(z^{*}\right)$ such that

$$
\gamma=\sum_{t=0}^{N} \gamma_{t}^{x}+\sum_{t=0}^{N-1} \gamma_{t}^{u}
$$

Proof of Claim: We know that the Clarke normal cone to any non-empty set is a closed and convex cone. Therefore, both $\left(N_{\Omega_{t}^{x}}^{C}\left(z^{*}\right)\right)_{t=0}^{N}$ and $\left(N_{\Omega_{t}^{u}}^{C}\left(z^{*}\right)\right)_{t=0}^{N-1}$ are sequences of closed and convex cones.

Consider the set $S:=\operatorname{co}\left(\left(\bigcup_{t=0}^{N} N_{\Omega_{t}^{x}}^{C}\left(z^{*}\right)\right) \cup\left(\bigcup_{t=0}^{N-1} N_{\Omega_{t}^{u}}^{C}\left(z^{*}\right)\right)\right)$. If $S$ is not closed, then by Theorem A.2 there exist vectors $\lambda_{t}^{x} \in N_{\Omega_{t}^{x}}^{C}\left(z^{*}\right)$, and $\lambda_{t}^{u} \in N_{\Omega_{t}^{u}}^{C}\left(z^{*}\right)$ for $t=$ $0, \ldots, N-1, \lambda_{N}^{x} \in N_{\Omega_{N}^{x}}^{C}\left(z^{*}\right)$, not all of them zero, such that $\sum_{t=0}^{N} \lambda_{t}^{x}+\sum_{t=0}^{N-1} \lambda_{t}^{u}=0$.

By the definition of the Clarke normal cone, if $\lambda_{t}^{x} \in N_{\Omega_{t}^{x}}^{C}\left(z^{*}\right)$, then for any vector $v_{t} \in T_{\Omega_{t}^{x}}^{C}\left(z^{*}\right)$ we have $\left\langle\lambda_{t}^{x}, v_{t}\right\rangle \leqslant 0$. For the set $\Omega_{t}^{x}$ defined in (5.5) $\pi_{s}^{x}\left(v_{t}\right)$ for $s \in$ 
$\{0, \ldots, N\} \backslash\{t\}$ and $\pi_{r}^{u}\left(v_{t}\right)$ for $r \in\{0, \ldots, N-1\}$ are arbitrary, and the corresponding coordinates in $\lambda_{t}^{x}$ are zeros. That is, $\pi_{s}^{x}\left(\lambda_{t}^{x}\right)=0$ for $s \in\{0, \ldots, N\} \backslash\{t\}$ and $\pi_{r}^{u}\left(\lambda_{t}^{x}\right)=0$ for $r \in\{0, \ldots, N-1\}$. Similarly, it follows that $\pi_{s}^{x}\left(\lambda_{t}^{u}\right)=0$ for $s \in\{0, \ldots, N\}$ and $\pi_{s}^{u}\left(\lambda_{t}^{u}\right)=0$ for $s \in\{0, \ldots, N-1\} \backslash\{t\}$. Therefore, $\lambda_{t}^{x}$ will be of form

$$
\begin{array}{ll}
\lambda_{t}^{x}=\left(0, \ldots, 0, \eta_{t}^{x}, 0, \ldots, 0\right) & \text { for } t=0, \ldots, N, \\
\lambda_{t}^{u}=\left(0, \ldots, 0, \eta_{t}^{u}, 0, \ldots, 0\right) & \text { for } t=0, \ldots, N-1,
\end{array}
$$

where $\eta_{t}^{x}:=\pi_{t}^{x}\left(\lambda_{t}^{x}\right), \eta_{t}^{u}:=\pi_{t}^{u}\left(\lambda_{t}^{u}\right)$ for $t=0, \ldots, N-1$, and $\eta_{N}^{x}:=\pi_{N}^{x}\left(\lambda_{N}^{x}\right)$. Hence, $\sum_{t=0}^{N} \lambda_{t}^{x}+\sum_{t=0}^{N-1} \lambda_{t}^{u}=0$ implies $\lambda_{t}^{x}=\lambda_{t}^{u}=\lambda_{N}^{x}=0$ for $0 \leqslant t \leqslant N-1$. This contradicts the non-triviality assertion on $\left(\lambda_{t}^{x}\right)_{t=0}^{N},\left(\lambda_{t}^{u}\right)_{t=0}^{N-1}$. Therefore, our assumption is not true which means $S$ is closed.

From Lemma B.2, $T_{\Omega}^{C}\left(z^{*}\right)=\left(\bigcap_{t=0}^{N} T_{\Omega_{t}^{x}}^{C}\left(z^{*}\right)\right) \cap\left(\bigcap_{t=0}^{N-1} T_{\Omega_{t}^{u}}^{C}\left(z^{*}\right)\right)$. Therefore, from Theorem A. 3 we see that

$$
N_{\Omega}^{C}\left(z^{*}\right)=\operatorname{cl}\left(\operatorname{co}\left(\left(\bigcup_{t=0}^{N} N_{\Omega_{t}^{x}}^{C}\right) \cup\left(\bigcup_{t=0}^{N-1} N_{\Omega_{t}^{u}}^{C}\right)\right)\right)=\operatorname{co}\left(\left(\bigcup_{t=0}^{N} N_{\Omega_{t}^{x}}^{C}\right) \cup\left(\bigcup_{t=0}^{N-1} N_{\Omega_{t}^{u}}^{C}\right)\right) .
$$

The assertion of the claim follows at once from the fact that $\left(N_{\Omega_{t}^{x}}^{C}\right)_{t=0}^{N}$ and $\left(N_{\Omega_{t}^{u}}^{C}\right)_{t=0}^{N-1}$ are sequences cones.

$\circ$ Consider, second, the set $\partial\left(\eta_{c} C(\cdot)+\left\langle\lambda^{f}, f_{d}(\cdot)\right\rangle+\left\langle\widehat{\eta^{x}}, \widehat{F}(\cdot)\right\rangle+\left\langle\widehat{\eta^{u}}, \widehat{G}(\cdot)\right\rangle\right)\left(z^{*}\right)$ in (5.10). The functions $C, \widehat{F}, \widehat{G}$ in (5.9) are continuously differentiable. Using Lemma B.1 we get,

$$
\begin{aligned}
& \partial\left(\eta_{c} C+\left\langle\lambda^{f}, f_{d}\right\rangle+\left\langle\widehat{\eta^{x}}, \widehat{F}\right\rangle+\left\langle\widehat{\eta^{u}}, \widehat{G}\right\rangle\right)\left(z^{*}\right)= \\
& \quad \eta_{c} \frac{\partial}{\partial z} C\left(z^{*}\right)+\partial\left\langle\lambda^{f}, f_{d}(\cdot)\right\rangle\left(z^{*}\right)+\left(\frac{\partial}{\partial z} \widehat{F}\left(z^{*}\right)\right)^{\top} \widehat{\eta^{x}}+\left(\frac{\partial}{\partial z} \widehat{G}\left(z^{*}\right)\right)^{\top} \widehat{\eta^{u}} .
\end{aligned}
$$

In other words, (5.10) can be simplified to

$$
0 \in \eta_{c} \frac{\partial}{\partial z} C\left(z^{*}\right)+\partial\left\langle\lambda^{f}, f_{d}(\cdot)\right\rangle\left(z^{*}\right)+\left(\frac{\partial}{\partial z} \widehat{F}\left(z^{*}\right)\right)^{\top} \widehat{\eta^{x}}+\left(\frac{\partial}{\partial z} \widehat{G}\left(z^{*}\right)\right)^{\top} \widehat{\eta^{u}}+N_{\Omega}^{C}\left(z^{*}\right)
$$

To characterize the elements of the set $\partial\left\langle\lambda^{f}, f_{d}(\cdot)\right\rangle\left(z^{*}\right)$, let us define the functions

$$
\begin{aligned}
& \Omega \ni z \longmapsto \tilde{f}_{t}(z):=\xi_{t+1}-f_{t}\left(\xi_{t}, \mu_{t}\right) \text { for } t=0, \ldots, N-1, \\
& \Omega \ni z \longmapsto h_{t}(z):=\left\langle\pi_{t}^{x}\left(\lambda^{f}\right), \tilde{f}_{t}(z)\right\rangle=\left\langle\eta_{t}^{f}, \tilde{f}_{t}(z)\right\rangle \in \mathbb{R} \text { for } t=0, \ldots, N-1, \\
& \Omega \ni z \longmapsto h(z):=\sum_{t=0}^{N-1} h_{t}(z)=\left\langle\lambda^{f}, f_{d}(z)\right\rangle \in \mathbb{R} .
\end{aligned}
$$

We immediately see that $\partial\left\langle\lambda^{f}, f_{d}(\cdot)\right\rangle\left(z^{*}\right)=\partial h\left(z^{*}\right)$.

If $\omega \in \partial h\left(z^{*}\right)$, then from the definition of generalized gradient (3.1) $\langle\omega, v\rangle \leqslant h^{\circ}\left(z^{*} ; v\right)$ for all $v \in \mathbb{R}^{v}$. Give that $f_{t}$ 's are regular functions ( cf. 4.1), the function $h$ defined above is also a regular function. Moreover, since $h$ is a regular function, $h$ has a directional derivative in each direction at $z^{*}$ and $h^{\circ}\left(z^{*} ; v\right)=\mathcal{D}_{v} h\left(z^{*}\right)=\sum_{t=0}^{N} \mathcal{D}_{v} h_{t}\left(z^{*}\right)$. Consequently,

$$
\langle\omega, v\rangle \leqslant \sum_{t=0}^{N} \mathcal{D}_{v} h_{t}\left(z^{*}\right) \quad \text { for all } v \in \mathbb{R}^{v} .
$$

Let $\omega \in \partial\left\langle\lambda^{f}, f_{d}(\cdot)\right\rangle\left(z^{*}\right)$ and $\lambda=\sum_{t=0}^{N} \lambda_{t}^{x}+\sum_{t=0}^{N-1} \lambda_{t}^{u} \in N_{\Omega}^{C}\left(z^{*}\right)$, where $\lambda_{t}^{x} \in$ $N_{\Omega_{t}^{x}}^{C}\left(z^{*}\right), \lambda_{t}^{u} \in N_{\Omega_{t}^{u}}^{C}\left(z^{*}\right)$, be vectors such that

$$
\eta_{c} \frac{\partial}{\partial z} C\left(z^{*}\right)+\omega+\left(\frac{\partial}{\partial z} \widehat{F}\left(z^{*}\right)\right)^{\top} \widehat{\eta^{x}}+\left(\frac{\partial}{\partial z} \widehat{G}\left(z^{*}\right)\right)^{\top} \widehat{\eta^{u}}+\sum_{t=0}^{N} \lambda_{t}^{x}+\sum_{t=0}^{N-1} \lambda_{t}^{u}=0,
$$


and a transposition leads to

$$
-\left(\eta_{c} \frac{\partial}{\partial z} C\left(z^{*}\right)+\left(\frac{\partial}{\partial z} \widehat{F}\left(z^{*}\right)\right)^{\top} \widehat{\eta^{x}}+\left(\frac{\partial}{\partial z} \widehat{G}\left(z^{*}\right)\right)^{\top} \widehat{\eta^{u}}+\sum_{t=0}^{N} \lambda_{t}^{x}+\sum_{t=0}^{N-1} \lambda_{t}^{u}\right)=\omega .
$$

Here $\omega \in \partial\left\langle\lambda^{f}, f_{d}(\cdot)\right\rangle\left(z^{*}\right)$ implies that $\langle\omega, v\rangle \leqslant \mathcal{D}_{v} h\left(z^{*}\right)$ for all $v \in \mathbb{R}^{v}$, and hence the inequality simplifies (5.14) to

$$
\begin{array}{r}
\left\langle-\left(\eta_{c} \frac{\partial}{\partial z} C\left(z^{*}\right)+\left(\frac{\partial}{\partial z} \widehat{F}\left(z^{*}\right)\right)^{\top} \widehat{\eta^{x}}+\left(\frac{\partial}{\partial z} \widehat{G}\left(z^{*}\right)\right)^{\top} \widehat{\eta^{u}}+\sum_{t=0}^{N} \lambda_{t}^{x}+\sum_{t=0}^{N-1} \lambda_{t}^{u}\right), v\right\rangle \\
\leqslant \sum_{t=0}^{N} \mathcal{D}_{v} h_{t}\left(z^{*}\right) \quad \text { for all } v \in \mathbb{R}^{v} .
\end{array}
$$

In other words we have established the following proposition:

Proposition 5.2. If $z^{*}$ is a solution of problem (5.9), then there exist a non-trivial vector $\left(\eta_{c}, \lambda^{f}, \widehat{\eta^{x}}, \widehat{\eta^{u}}\right) \in\{0,1\} \times\left(\mathbb{R}^{d N}\right)^{\star} \times\left(\mathbb{R}^{v_{x}}\right)^{\star} \times\left(\mathbb{R}^{v_{u}}\right)^{\star}$, vector $\lambda_{t}^{x} \in N_{\Omega_{t}^{x}}^{C}\left(z^{*}\right)$ for each $t=0, \ldots, N$, and vector $\lambda_{t}^{u} \in N_{\Omega_{t}^{u}}^{C}\left(z^{*}\right)$ for each $t=0, \ldots, N-1$ such that (5.15) holds true, where $h_{t}$ is as defined in (5.13).

The inequality (5.15) obtained is a necessary condition for the solutions of the equivalent optimization problem (5.9).

\subsection{Step (III). Projecting the condition in equation (5.15) to the original factor spaces:}

We use Proposition 5.2 to arrive at a set of necessary conditions for a solution of the optimal control problem (2.2). It may be observed, in particular, that the non-negativity condition $(\mathrm{C}-\mathrm{i})$ in the main result follows directly from (5.10).

For some $t \in\{0, \ldots, N\}$, choose a vector $v_{t}^{x} \in \mathbb{R}^{d}$ and define

$$
\tilde{v}:=\left(0, \ldots, v_{t}^{x}, 0, \ldots, 0\right) \in \mathbb{R}^{v} .
$$

The projections of $\tilde{v}(5.2)$ are

$$
\pi_{i}^{x}(\tilde{v})=\left\{\begin{array}{ll}
v_{t}^{x} & \text { for } i=t, \\
0 & \text { otherwise, }
\end{array} \quad \text { and } \quad \pi_{i}^{u}(\tilde{v})=0 \quad \text { for } i=0, \ldots, N-1\right.
$$

Substituting $v=\tilde{v}$ in (5.15), we get

$$
\begin{array}{r}
\left\langle-\left(\eta_{c} \frac{\partial}{\partial z} C\left(z^{*}\right)+\left(\frac{\partial}{\partial z} \widehat{F}\left(z^{*}\right)\right)^{\top} \widehat{\eta^{x}}+\left(\frac{\partial}{\partial z} \widehat{G}\left(z^{*}\right)\right)^{\top} \widehat{\eta^{u}}+\sum_{i=0}^{N} \lambda_{i}^{x}+\sum_{i=0}^{N-1} \lambda_{i}^{u}\right), \tilde{v}\right\rangle \\
\leqslant \sum_{i=0}^{N} \mathcal{D}_{\tilde{v}} h_{i}\left(z^{*}\right) .
\end{array}
$$

Let us look at each term individually, starting from the left, in the above inequality.

- The first term corresponds to the cost function $(C)$ of the optimization problem. From the definition (5.3) of $C$, its gradient is

$$
\begin{aligned}
& \frac{\partial}{\partial z} C\left(z^{*}\right)=
\end{aligned}
$$

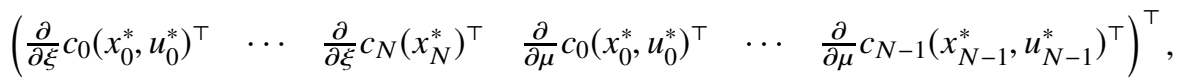


and therefore,

$$
\left\langle\eta_{c} \frac{\partial}{\partial z} C\left(z^{*}\right), \tilde{v}\right\rangle=\eta_{c}\left(\sum_{i=0}^{N}\left\langle\frac{\partial}{\partial \xi} c_{i}\left(x_{i}^{*}, u_{i}^{*}\right), \pi_{i}^{x}(\tilde{v})\right\rangle+\sum_{i=0}^{N-1}\left\langle\frac{\partial}{\partial \mu} c_{i}\left(x_{i}^{*}, u_{i}^{*}\right), \pi_{i}^{u}(\tilde{v})\right\rangle\right) .
$$

Substituting the projections of $\tilde{v}$ from (5.17) gives

$$
\left\langle\eta_{c} \frac{\partial}{\partial z} C\left(z^{*}\right), \tilde{v}\right\rangle=\left\langle\eta_{c} \frac{\partial}{\partial \xi} c_{t}\left(x_{t}^{*}, u_{t}^{*}\right), \pi_{t}^{x}(\tilde{v})\right\rangle=\left\langle\eta_{c} \frac{\partial}{\partial \xi} c_{t}\left(x_{t}^{*}, u_{t}^{*}\right), v_{t}^{x}\right\rangle .
$$

- The second term in (5.18) corresponds to the constraints on the state trajectory (in particular, to the function $F$,) of the original optimal control problem, equivalently represented by the function $\widehat{F}$ in $\mathbb{R}^{v}$. Recalling the definition (5.7) of $\widehat{F}$, we see that

$$
\widehat{F}\left(\xi_{0}, \cdots, \xi_{N}, \mu_{0}, \cdots, \mu_{N-1}\right)=F\left(\xi_{0}, \cdots, \xi_{N}\right) \in \mathbb{R}^{v_{x}} .
$$

Let $\widehat{F}=\left(\begin{array}{lll}\widehat{F}_{1} & \cdots & \widehat{F}_{v_{x}}\end{array}\right)^{\top}$. Its gradient is given by

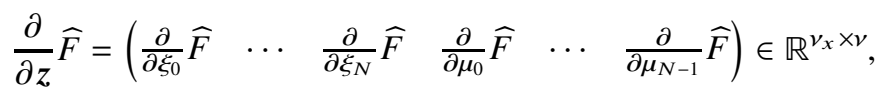

where

$$
\begin{aligned}
& \frac{\partial}{\partial \xi_{i}} \widehat{F}=\left(\begin{array}{ccc}
\frac{\partial}{\partial \xi_{i}^{1}} \widehat{F}_{1} & \cdots & \frac{\partial}{\partial \xi_{i}^{d}} \widehat{F}_{1} \\
\vdots & \ddots & \vdots \\
\frac{\partial}{\partial \xi_{i}^{1}} \widehat{F}_{v_{x}} & \cdots & \frac{\partial}{\partial \xi_{i}^{d}} \widehat{F}_{v_{x}}
\end{array}\right) \in \mathbb{R}^{v_{x} \times d} \text { and } \\
& \frac{\partial}{\partial \mu_{i}} \widehat{F}=\left(\begin{array}{ccc}
\frac{\partial}{\partial \mu_{i}^{1}} \widehat{F}_{1} & \cdots & \frac{\partial}{\partial \mu_{i}^{m}} \widehat{F}_{1} \\
\vdots & \ddots & \vdots \\
\frac{\partial}{\partial \mu_{i}^{1}} \widehat{F}_{v_{x}} & \cdots & \frac{\partial}{\partial \mu_{i}^{m}} \widehat{F}_{v_{x}}
\end{array}\right) \in \mathbb{R}^{v_{x} \times m}
\end{aligned}
$$

From the definition of $\widehat{F}$ we see at once that $\frac{\partial}{\partial \mu_{i}} \widehat{F}=0$ for $i=0, \ldots, N-1$ and $\frac{\partial}{\partial \xi_{i}} \widehat{F}=\frac{\partial}{\partial \xi_{i}} F$ for $i=0, \ldots, N$, which shows that

$$
\frac{\partial}{\partial z} \widehat{F}=\left(\begin{array}{llll}
\frac{\partial}{\partial \xi_{0}} F & \cdots & \frac{\partial}{\partial \xi_{N}} F & \mathbf{0}_{v_{x} \times m N}
\end{array}\right) \in \mathbb{R}^{v_{x} \times v} .
$$

Moreover, from the definition (2.2-d) of $F$, we see that $\frac{\partial}{\partial \xi_{i}} F=\frac{\partial}{\partial \xi} \widetilde{F}_{i}$ for $i=0, \ldots, N$. This implies, for a vector $\widehat{\eta^{x}} \in \mathbb{R}^{v_{x}}$,

$$
\left(\frac{\partial}{\partial z} \widehat{F}\right)^{\top} \widehat{\eta^{x}}=\left(\begin{array}{c}
\left(\frac{\partial}{\partial \xi} \widetilde{F}_{0}\right)^{\top} \widehat{\eta^{x}} \\
\vdots \\
\left(\frac{\partial}{\partial \xi} \widetilde{F}_{N}\right)^{\top} \widehat{\eta^{x}} \\
\mathbf{0}_{m N \times 1}
\end{array}\right) \in \mathbb{R}^{v}
$$

Therefore, the second term in (5.18) for the projection (5.17) of given $\tilde{v}$ is

$$
\left\langle\left(\frac{\partial}{\partial z} \widehat{F}\left(z^{*}\right)\right)^{\top} \widehat{\eta^{x}}, \tilde{v}\right\rangle=\sum_{i=0}^{N}\left\langle\left(\frac{\partial}{\partial \xi} \widetilde{F}_{i}\left(x_{i}^{*}\right)\right)^{\top} \widehat{\eta^{x}}, \pi_{i}^{x}(\tilde{v})\right\rangle=\left\langle\left(\frac{\partial}{\partial \xi} \widetilde{F}_{t}\left(x_{t}^{*}\right)\right)^{\top} \widehat{\eta^{x}}, \tilde{v}_{t}^{x}\right\rangle .
$$

- The third term in (5.18) corresponds to the frequency constraints on the control trajectory (in particular, to the function $G$,) of the original optimal control problem, equivalently represented by the function $\widehat{G}$ in $\mathbb{R}^{v}$. Recalling the definition (5.8) of $\widehat{G}$ we see that

$$
\widehat{G}\left(\xi_{0}, \ldots, \xi_{N}, \mu_{0}, \ldots, \mu_{N-1}\right)=G\left(\mu_{0}, \ldots, \mu_{N-1}\right) \in \mathbb{R}^{v_{u}} .
$$


Say $\widehat{G}=\left(\begin{array}{lll}\widehat{G}_{1} & \cdots & \widehat{G}_{v_{u}}\end{array}\right)^{\top}$. Its gradient is given by

$$
\frac{\partial}{\partial z} \widehat{G}=\left(\begin{array}{llllll}
\frac{\partial}{\partial \xi_{0}} \widehat{G} & \cdots & \frac{\partial}{\partial \xi_{N}} \widehat{G} \quad \frac{\partial}{\partial \mu_{0}} \widehat{G} \quad \cdots \quad \frac{\partial}{\partial \mu_{N-1}} \widehat{G}
\end{array}\right) \in \mathbb{R}^{v_{u} \times v},
$$

where

$$
\begin{gathered}
\frac{\partial}{\partial \xi_{i}} \widehat{G}=\left(\begin{array}{ccc}
\frac{\partial}{\partial \xi_{i}^{1}} \widehat{G}_{1} & \cdots & \frac{\partial}{\partial \xi_{i}^{d}} \widehat{G}_{1} \\
\vdots & \ddots & \vdots \\
\frac{\partial}{\partial \xi_{i}^{1}} \widehat{G}_{v_{u}} & \cdots & \frac{\partial}{\partial \xi_{i}^{d}} \widehat{G}_{v_{u}}
\end{array}\right) \in \mathbb{R}^{v_{u} \times d} \text { and } \\
\frac{\partial}{\partial \mu_{i}} \widehat{G}=\left(\begin{array}{ccc}
\frac{\partial}{\partial \mu_{i}^{1}} \widehat{G}_{1} & \cdots & \frac{\partial}{\partial \mu_{i}^{m}} \widehat{G}_{1} \\
\vdots & \ddots & \vdots \\
\frac{\partial}{\partial \mu_{i}^{1}} \widehat{G}_{v_{u}} & \cdots & \frac{\partial}{\partial \mu_{i}^{m}} \widehat{G}_{v_{u}}
\end{array}\right) \in \mathbb{R}^{v_{u} \times m} .
\end{gathered}
$$

We see at once that $\frac{\partial}{\partial \xi_{i}} \widehat{G}=0$ for all $i=0, \ldots N$ and $\frac{\partial}{\partial \mu_{i}} \widehat{G}=\frac{\partial}{\partial \mu_{i}} G$ for all $i=0, \ldots N-1$,. Therefore,

$$
\frac{\partial}{\partial z} \widehat{G}=\left(\begin{array}{llll}
\mathbf{0}_{v_{u} \times d(N+1)} & \frac{\partial}{\partial \mu_{0}} G & \cdots & \frac{\partial}{\partial \mu_{N-1}} G
\end{array}\right) \in \mathbb{R}^{v_{u} \times v} .
$$

Moreover, from the definition (2.2-e) of $G, \frac{\partial}{\partial \mu_{i}} G=\frac{\partial}{\partial \mu} \widetilde{G}_{i}$ for $i=0, \ldots, N-1$. This implies, for a vector $\widehat{\eta^{u}} \in \mathbb{R}^{v_{u}}$,

$$
\left(\frac{\partial}{\partial z} \widehat{G}\right)^{\top} \widehat{\eta^{u}}=\left(\begin{array}{c}
\mathbf{0}_{d(N+1) \times 1} \\
\left(\frac{\partial}{\partial \mu} \widetilde{G}_{0}\right)^{\top} \widehat{\eta^{u}} \\
\vdots \\
\left(\frac{\partial}{\partial \mu} \widetilde{G}_{N-1}\right)^{\top} \widehat{\eta^{u}}
\end{array}\right) \in \mathbb{R}^{v}
$$

Therefore, the third term in (5.18) for the projection (5.17) of given $\tilde{v}$ can be written as

$$
\left\langle\left(\frac{\partial}{\partial z} \widehat{G}\left(z^{*}\right)\right)^{\top} \widehat{\eta^{u}}, \tilde{v}\right\rangle=\sum_{i=0}^{N}\left\langle 0, \pi_{i}^{x}(\tilde{v})\right\rangle+\sum_{i=0}^{N-1}\left\langle\left(\frac{\partial}{\partial \mu} \widetilde{G}_{i}\left(u_{t}^{*}\right)\right)^{\top} \widehat{\eta^{u}}, \pi_{i}^{u}(\tilde{v})\right\rangle=0 .
$$

- The fourth and fifth term in (5.18) correspond to the point-wise constraints on the states and the control actions of the original optimal control problem, respectively. For the equivalent optimization problem 5.9, they are multipliers corresponding to the state constraints such that each $\lambda_{t}^{x} \in N_{\Omega_{t}^{x}}^{C}\left(z^{*}\right), \lambda_{t}^{u} \in N_{\Omega_{t}^{u}}^{C}\left(z^{*}\right)$, and from (5.11) we see that

$$
\begin{gathered}
\lambda_{t}^{x}=\left(0, \ldots, 0, \eta_{t}^{x}, 0, \ldots, 0\right) \quad \text { for } t=0, \ldots, N \\
\lambda_{t}^{u}=\left(0, \ldots, 0, \eta_{t}^{u}, 0, \ldots, 0\right) \quad \text { for } t=0, \ldots, N-1 .
\end{gathered}
$$

Therefore, for the given $\tilde{v}$ we can write

$$
\left\langle\lambda_{s}^{x}, \tilde{v}\right\rangle=\left\{\begin{array}{ll}
\left\langle\eta_{t}^{x}, v_{t}^{x}\right\rangle & \text { for } s=t, \\
0 & \text { otherwise },
\end{array} \quad\left\langle\lambda_{s}^{u}, \tilde{v}\right\rangle=0 \text { for } s=0, \ldots, N-1,\right.
$$

The fourth and fifth terms in (5.18) become

$$
\left\langle\left(\sum_{i=0}^{N} \lambda_{i}^{x}+\sum_{i=0}^{N-1} \lambda_{i}^{u}\right), \tilde{v}\right\rangle=\left\langle\eta_{t}^{x}, v_{t}^{x}\right\rangle
$$

- Finally, the term on the right hand side of (5.18) is a sum of the directional derivatives of the functions $h_{i}$ along the direction $\tilde{v}$. The functions $h_{i}$, defined in (5.13), correspond to the dynamics of the original system at the $i^{\text {th }}$ time instance. Recall the definitions 
(3.1) and (5.13) of the directional derivative and the functions $\left(h_{i}\right)_{i=0}^{N-1}$, respectively, and observe that

$$
\mathcal{D}_{\tilde{v}} h_{i}\left(z^{*}\right)=\lim _{\theta \downarrow 0} \frac{h_{i}\left(z^{*}+\theta \tilde{v}\right)-h_{i}\left(z^{*}\right)}{\theta} \text { and } h_{i}(z)=\left\langle\eta_{i}^{f}, \pi_{i+1}^{x}(z)-f_{i}\left(\pi_{i}^{x}(z), \pi_{i}^{u}(z)\right)\right\rangle .
$$

Therefore the directional derivative of each $h_{i}$ along $\tilde{v}$ is given by

$$
\mathcal{D}_{\tilde{v}} h_{i}\left(z^{*}\right)=\lim _{\theta \downarrow 0} \frac{\left\langle\eta_{i}^{f}, \theta \pi_{i+1}^{x}(\tilde{v})-f_{i}\left(x_{i}^{*}+\theta \pi_{i}^{x}(\tilde{v}), u_{i}^{*}+\theta \pi_{i}^{u}(\tilde{v})\right)+f_{i}\left(x_{i}^{*}, u_{i}^{*}\right)\right\rangle}{\theta} .
$$

From the projections of $\tilde{v}$ given in (5.17), the above equation can be simplified to

$$
\mathcal{D}_{\tilde{v}} h_{i}\left(z^{*}\right)= \begin{cases}-\left\langle\eta_{t}^{f}, \mathcal{D}_{v_{t}^{x}} f_{t}\left(\cdot, u_{t}^{*}\right)\left(x_{t}^{*}\right)\right\rangle & \text { for } i=t, \\ \left\langle\eta_{t-1}^{f}, v_{t}^{x}\right\rangle & \text { for } i=t-1, \\ 0 & \text { otherwise. }\end{cases}
$$

In other words, the term on the right hand side of (5.18) is given by

$$
\sum_{i=0}^{N} \mathcal{D}_{\tilde{v}} h_{i}\left(z^{*}\right)= \begin{cases}\mathcal{D}_{\tilde{v}} h_{0}\left(z^{*}\right) & \text { for } t=0, \\ \mathcal{D}_{\tilde{v}} h_{t-1}\left(z^{*}\right)+\mathcal{D}_{\tilde{v}} h_{t}\left(z^{*}\right) & \text { for } t=1, \ldots, N-1, \\ \mathcal{D}_{\tilde{v}} h_{N-1}\left(z^{*}\right) & \text { for } t=N,\end{cases}
$$

which leads to

$$
\sum_{i=0}^{N} \mathcal{D}_{\tilde{v}} h_{i}\left(z^{*}\right)= \begin{cases}-\left\langle\eta_{0}^{f}, \mathcal{D}_{v_{0}^{x}} f_{0}\left(\cdot, u_{0}^{*}\right)\left(x_{0}^{*}\right)\right\rangle & \text { for } t=0, \\ \left\langle\eta_{t-1}^{f}, v_{t}^{x}\right\rangle-\left\langle\eta_{t}^{f}, \mathcal{D}_{v_{t}^{x}} f_{t}\left(\cdot, u_{t}^{*}\right)\left(x_{t}^{*}\right)\right\rangle & \text { for } t=1, \ldots, N-1, \\ \left\langle\eta_{N-1}^{f}, v_{N}^{x}\right\rangle & \text { for } t=N .\end{cases}
$$

Substituting each term in (5.18) using equations (5.19), (5.20), (5.21), (5.22) and (5.23), we arrive the following:

$\circ$ For $t=0$,

$$
\left\langle-\eta_{c} \frac{\partial}{\partial \xi} c_{0}\left(x_{0}^{*}, u_{0}^{*}\right)-\left(\frac{\partial}{\partial \xi} \widetilde{F}_{0}\left(x^{*}\right)\right)^{\top} \widehat{\eta^{x}}-\eta_{0}^{x}, v_{0}^{x}\right\rangle \leqslant-\left\langle\eta_{0}^{f}, \mathcal{D}_{v_{0}^{x}} f_{0}\left(\cdot, u_{0}^{*}\right)\left(x_{0}^{*}\right)\right\rangle .
$$

Since $v_{0}^{x} \in \mathbb{R}^{d}$ is an arbitrary vector in $\mathbb{R}^{d}$, this means

$$
\left\langle-\eta_{c} \frac{\partial}{\partial \xi} c_{0}\left(x_{0}^{*}, u_{0}^{*}\right)-\left(\frac{\partial}{\partial \xi} \widetilde{F}_{0}\left(x^{*}\right)\right)^{\top} \widehat{\eta^{x}}-\eta_{0}^{x}, y\right\rangle \leqslant-\left\langle\eta_{0}^{f}, \mathcal{D}_{y} f_{0}\left(\cdot, u_{0}^{*}\right)\left(x_{0}^{*}\right)\right\rangle
$$

for all $y \in \mathbb{R}^{d}$.

○ For $t \in\{1, \ldots, N-1\}$,

$$
\left\langle-\eta_{c} \frac{\partial}{\partial \xi} c_{t}\left(x_{t}^{*}, u_{t}^{*}\right)-\left(\frac{\partial}{\partial \xi} \widetilde{F}_{t}\left(x^{*}\right)\right)^{\top} \widehat{\eta}^{x}-\eta_{t}^{x}, v_{t}^{x}\right\rangle \leqslant\left\langle\eta_{t-1}^{f}, v_{t}^{x}\right\rangle-\left\langle\eta_{t}^{f}, \mathcal{D}_{v_{t}^{x}} f_{t}\left(\cdot, u_{t}^{*}\right)\left(x_{t}^{*}\right)\right\rangle .
$$

Since $v_{t}^{x} \in \mathbb{R}^{d}$ is an arbitrary vector in $\mathbb{R}^{d}$, this means

$$
\left\langle-\eta_{c} \frac{\partial}{\partial \xi} c_{t}\left(x_{t}^{*}, u_{t}^{*}\right)-\left(\frac{\partial}{\partial \xi} \widetilde{F}_{t}\left(x^{*}\right)\right)^{\top} \widehat{\eta^{x}}-\eta_{t}^{x}, y\right\rangle \leqslant\left\langle\eta_{t-1}^{f}, y\right\rangle-\left\langle\eta_{t}^{f}, \mathcal{D}_{y} f_{t}\left(\cdot, u_{t}^{*}\right)\left(x_{t}^{*}\right)\right\rangle .
$$

○ For $t=N$

$$
\left\langle-\eta_{c} \frac{\partial}{\partial \xi} c_{N}\left(x_{N}^{*}, u_{N}^{*}\right)-\left(\frac{\partial}{\partial \xi} \widetilde{F}_{N}\left(x^{*}\right)\right)^{\top} \widehat{\eta^{x}}-\eta_{N}^{x}, v_{N}^{x}\right\rangle \leqslant-\left\langle\eta_{N-1}^{f}, v_{N}^{x}\right\rangle
$$


Since $v_{N}^{x} \in \mathbb{R}^{d}$ is an arbitrary vector in $\mathbb{R}^{d}$, this means

$$
\left\langle-\eta_{c} \frac{\partial}{\partial \xi} c_{N}\left(x_{N}^{*}, u_{N}^{*}\right)-\left(\frac{\partial}{\partial \xi} \widetilde{F}_{N}\left(x^{*}\right)\right)^{\top} \widehat{\eta^{x}}-\eta_{N}^{x}, y\right\rangle \leqslant-\left\langle\eta_{N-1}^{f}, y\right\rangle \text { for all } y \in \mathbb{R}^{d},
$$

which implies

$$
-\eta_{c} \frac{\partial}{\partial \xi} c_{N}\left(x_{N}^{*}\right)-\left(\frac{\partial}{\partial \xi} \widetilde{F}_{N}\left(x^{*}\right)\right)^{\top} \widehat{\eta^{x}}-\eta_{N}^{x}+\eta_{N-1}^{f}=0 .
$$

Similarly, if we put $v=\hat{v}=\left(0, \cdots, 0, v_{t}^{u}, 0, \cdots, 0\right) \in \mathbb{R}^{v}$ for some $t \in\{0, \cdots, N-1\}$, in (5.15), then for all $v_{t}^{u} \in \mathbb{R}^{m}$, each term of (5.15) equivalently translates to

$$
\left\{\begin{array}{l}
\left\langle\eta_{c} \frac{\partial}{\partial z} C\left(z^{*}\right), \hat{v}\right\rangle=\left\langle\eta_{c} \frac{\partial}{\partial \mu} c_{t}\left(x_{t}^{*}, u_{t}^{*}\right), \pi_{t}^{u}(\hat{v})\right\rangle=\left\langle\eta_{c} \frac{\partial}{\partial \mu} c_{t}\left(x_{t}^{*}, u_{t}^{*}\right), v_{t}^{u}\right\rangle, \\
\left\langle\left(\frac{\partial}{\partial z} \widehat{F}\left(z^{*}\right)\right)^{\top} \widehat{\eta^{x}}, \hat{v}\right\rangle=\sum_{i=0}^{N}\left\langle\left(\frac{\partial}{\partial \xi} \widetilde{F}_{i}\left(u_{t}^{*}\right)\right)^{\top} \widehat{\eta^{x}}, \pi_{i}^{x}(\hat{v})\right\rangle+\sum_{i=0}^{N-1}\left\langle 0, \pi_{i}^{u}(\hat{v})\right\rangle=0, \\
\left\langle\left(\frac{\partial}{\partial z} \widehat{G}\left(z^{*}\right)\right)^{\top} \widehat{\eta^{u}}, \hat{v}\right\rangle=\sum_{i=0}^{N-1}\left\langle\left(\frac{\partial}{\partial \mu} \widetilde{G}_{i}\left(u_{i}^{*}\right)\right)^{\top} \widehat{\eta^{u}}, \pi_{i}^{u}(\hat{v})\right\rangle=\left\langle\left(\frac{\partial}{\partial \mu} \widetilde{G}_{t}\left(u_{t}^{*}\right)\right)^{\top} \widehat{\eta^{u}}, v_{t}^{u}\right\rangle, \\
\left\langle\left(\sum_{i=0}^{N} \lambda_{i}^{x}+\sum_{i=0}^{N-1} \lambda_{i}^{u}\right), \hat{v}\right\rangle=\left\langle\eta_{t}^{u}, v_{t}^{u}\right\rangle, \\
\mathcal{D}_{\hat{v}} h_{t}\left(z^{*}\right)=-\left\langle\eta_{t}^{f}, \mathcal{D}_{v_{t}^{u}} f_{t}\left(x_{t}^{*}, \cdot\right)\left(u_{t}^{*}\right)\right\rangle .
\end{array}\right.
$$

Therefore, the inequality $(5.15)$ can be rewritten as

$$
\begin{array}{r}
\left\langle-\frac{\partial}{\partial \mu} c_{t}\left(x_{t}^{*}, u_{t}^{*}\right)-\frac{\partial}{\partial \mu} \widetilde{G}_{t}\left(x_{t}^{*}, u_{t}^{*}\right)-\eta_{t}^{u}, v_{t}^{u}\right\rangle \\
\text { for all } v_{t}^{u} \in-\left\langle\eta_{t}^{f}, \mathbb{R}^{m} \text { for } t=0, \ldots, N-1 .\right.
\end{array}
$$

Observe from (4.1) that for $t=0, \ldots, N-1$,

for all $y \in \mathbb{R}^{d}$ we have,

$$
\begin{aligned}
\mathcal{D}_{y} H^{\eta_{c}, \widehat{\eta^{x}}, \widehat{\eta^{u}}}\left(\eta_{t}^{f}, t, \cdot u_{t}^{*}\right)\left(x_{t}^{*}\right)= & \left\langle-\eta_{c} \frac{\partial}{\partial \xi} c_{t}\left(x_{t}^{*}, u_{t}^{*}\right)-\left(\frac{\partial}{\partial \xi} \widetilde{F}_{t}\left(x^{*}\right)\right)^{\top} \widehat{\eta^{x}}, y\right\rangle \\
& +\left\langle\eta_{t}^{f}, \mathcal{D}_{y} f_{t}\left(\cdot, u_{t}^{*}\right)\left(x_{t}^{*}\right)\right\rangle,
\end{aligned}
$$

and for all $p \in \mathbb{R}^{m}$,

$$
\begin{aligned}
\mathcal{D}_{p} H^{\eta_{c}, \widehat{\eta^{x}}, \widehat{\eta^{u}}}\left(\eta_{t}^{f}, t, x_{t}^{*}, \cdot\right)\left(u_{t}^{*}\right)= & \left\langle-\eta_{c} \frac{\partial}{\partial \mu} c_{t}\left(x_{t}^{*}, u_{t}^{*}\right)-\left(\frac{\partial}{\partial \mu} \widetilde{G}_{t}\left(u^{*}\right)\right)^{\top} \widehat{\eta^{u}}, p\right\rangle . \\
& +\left\langle\eta_{t}^{f}, \mathcal{D}_{p} f_{t}\left(x_{t}^{*}, \cdot\right)\left(u_{t}^{*}\right)\right\rangle
\end{aligned}
$$

Substituting (5.29) and (5.30) in (5.25) and (5.28) respectively, we get the adjoint equation and the Hamiltonian maximization condition in (C-iii), (C-v) in Theorem 4.2, respectively. Clearly, the state equations in (C-iii) follow from the definition of the Hamiltonian. Moreover, the non-negativity condition (C-i) follows from (5.10). Finally, the non-triviality condition (C-ii) follows from the non-triviality of $\left(\eta_{c}, \lambda^{f}, \widehat{\eta^{x}}, \widehat{\eta^{u}}\right)$ in $(5.10)$ and $\lambda^{f}=\left(\eta_{0}^{f}, \ldots, \eta_{N-1}^{f}\right)$ in (5.10). 


\section{Numerical EXPERIMENTS}

6.1. Example 1. Inverted Pendulum on a Cart System: In our first example, we consider an optimal control problem with constraints on frequency components of control action for a linear discrete time model of pendulum on a cart system. First we design an optimal control for the problem via classical PMP and in order to satisfy constraints on frequency of control action we filter out the corresponding frequency components. Next, we use the necessary conditions proposed in Corollary 4.9 to design an optimal control. The control actions obtained via both the methods are fed to the system and the corresponding system trajectories are observed.

The specifications of the system considered are given below:

\begin{tabular}{l|l}
\hline Parameter & Value \\
\hline Mass of cart $(M)$ & $2.5 \mathrm{~kg}$ \\
Mass of pendulum $(m)$ & $0.6 \mathrm{~kg}$ \\
Half-length of pendulum $(l)$ & $0.25 \mathrm{~cm}$ \\
Range of cart track $(-L$ to $L)$ & $L=0.5 \mathrm{~cm}$ \\
Acceleration due to gravity $(g)$ & $9.8 \mathrm{~m} \mathrm{~s}^{-2}$ \\
\hline
\end{tabular}

TABLE 1. System Specifications

The continuous time linearized model of the pendulum on a cart system is described by the following differential equation:

$$
\dot{x}=A_{c} x+B_{c} u,
$$

where $x \in \mathbb{R}^{4}$,

$$
A_{c}=\left(\begin{array}{cccc}
0 & 0 & 1 & 0 \\
0 & 0 & 0 & 1 \\
0 & -\frac{(m l)^{2} g}{\left(J+m l^{2}\right)\left(m+M-\left(m^{2} l^{2} / J+m l^{2}\right)\right)} & 0 & 0 \\
0 & \frac{m g l(m+M)}{\left(J+m l^{2}\right)\left(m+M-\left(m^{2} l^{2} / J+m l^{2}\right)\right)} & 0 & 0
\end{array}\right) \quad \text { and } \quad B_{c}=\left(\begin{array}{c}
0 \\
0 \\
\frac{1}{\left(m+M-\left(m^{2} l^{2} / J+m l^{2}\right)\right)} \\
\frac{\left(J+m l^{2}\right)\left(m+M-\left(m^{2} l^{2} / J+m l^{2}\right)\right)}{(m+M}
\end{array}\right) .
$$

We obtain the discretized model using a zero-order-hold technique; assuming constant input over the sampling time $T_{s}=0.1 \mathrm{~s}$ and the corresponding discrete time linearized systems dynamics is governed by the following difference equation:

$$
x_{t+1}=A x_{t}+B u_{t},
$$

where

$\circ \mathbb{R}^{4} \ni x_{t}:=\left(x_{t}^{(1)}, x_{t}^{(2)}, x_{t}^{(3)}, x_{t}^{(4)}\right)$ is the state vector with $x_{t}^{(1)}$ denoting the position of the cart, $x_{t}^{(2)}$ denotes the angle of the pendulum with respect to y-axis, $x_{t}^{(3)}$ the linear velocity of the cart, and $x_{t}^{(4)}$ denotes the angular velocity of the pendulum, at $t^{\text {th }}$ time instant,

- control $u_{t} \in \mathbb{R}$ represents the force applied to the cart at $t^{\text {th }}$ instant,

$\circ$ and $A \in \mathbb{R}^{4 \times 4}, B \in \mathbb{R}^{4}$ are the system matrices.

Problem Description: Our objective is to drive the cart from a given initial condition $\bar{x}$ to a specified final position $x_{\mathrm{f}}$ in $N$ time steps; while minimizing the control effort and satisfying the following constraints

$$
\begin{cases}\left|x_{t}^{(i)}\right| \leqslant x_{\mathrm{b}}^{(i)} & \text { for } i=1, \ldots, 4, \text { and } t=0, \ldots, N, \\ u_{t} \leqslant u_{\mathrm{b}} & \text { for } t=0, \ldots, N-1, \\ \hat{u}_{i}=0 & \text { for } i \notin \mathcal{F}, \\ x_{0}=\bar{x}, \quad x_{N}=x_{\mathrm{f}}, & \end{cases}
$$


where the bounds $x_{\mathrm{b}}^{(i)}, u_{\mathrm{b}}$ on states and control represent pointwise constraints and the set of allowable frequencies $\mathcal{F}$ represents frequency constraints. The optimal control problem is written as

$$
\begin{array}{ll}
\underset{\left(u_{t}\right)_{t=0}^{N-1}}{\operatorname{minimize}} & \sum_{t=0}^{N-1} u_{t}^{2} \\
\text { subject to } & \{\text { dynamics (6.2) and constraints (6.3). }
\end{array}
$$

For this problem, we have assumed state bounds $\left(x_{\mathrm{b}}^{(1)}, x_{\mathrm{b}}^{(2)}, x_{\mathrm{b}}^{(3)}, x_{\mathrm{b}}^{(4)}\right)=\left(0.2, \frac{20 \pi}{180}, 15,30\right)$, control bound $u_{\mathrm{b}}=5 \mathrm{~N}$, length of horizon $N=240$ and the set $\mathcal{F}:=\{1, \ldots, 96,144, \ldots, 240\}$ corresponding to the low pass filter with cut off frequency $\frac{4 \pi}{5}$.

Using the first order necessary conditions proposed in Corollary 4.9, we obtain a two point boundary value problem. The solution of the boundary value problem is an optimal control, say $u_{\mathrm{p}}$ and denote its frequency components by $\hat{u}_{\mathrm{p}}$. Consider an optimal control obtained through the classical discrete time PMP incorporating only pointwise constraints on control and state (i.e., neglecting the frequency constraints on the control profile). The control so obtained is then passed through a proper filter in order to satisfy the frequency constraints on control profile, say $u_{\mathrm{f}}$. Let $\hat{u}_{\mathrm{f}}$ denote the frequency components of $u_{\mathrm{f}}$.

The time domain and frequency domain profiles of both the control actions are shown in Figure 3. It is evident that the control $u_{\mathrm{p}}$ satisfies the constraints in the problem 6.3.

Both the controls $u_{\mathrm{p}}$ and $u_{\mathrm{f}}$ are fed to the system (6.2) and the phase portraits corresponding to the initial condition $\bar{x}$ are shown in Fig. $3 \mathrm{C}$ and Fig. 3D. It is clear from the plots that the control via frequency constrained PMP $u_{\mathrm{p}}$ respects pointwise state constraints and end point states are also attained. On the other hand the filtered control $u_{\mathrm{f}}$, because of removal of certain frequency components, is unable to maintain the pointwise state constraints and does not reach the final state.

Unlike the previous example, the next two examples incorporate non-smooth features in the system dynamics. In each case we present the necessary conditions of Theorem 4.1 for the particular case.

6.2. Example 2. In our second example, we consider a discrete time system [GP17, Example 5.8, p. 98]; governed by the following difference equation

$$
x_{t+1}=f\left(x_{t}, u_{t}\right) \quad \text { for } t=0, \ldots, N-1,
$$

where $x_{t} \in \mathbb{R}^{2}, u_{t} \in \mathbb{R}$, and $\mathbb{R}^{2} \times \mathbb{R} \ni(\xi, \mu) \longmapsto f(\xi, \mu):=\left(\begin{array}{c}\xi^{(1)}(1-\mu) \\ \|\xi\| \mu\end{array}\right) \in \mathbb{R}^{2}$. Our objective is to characterize a solution of the following problem

$$
\begin{array}{ll}
\underset{\left(u_{t}\right)_{t=0}^{N-1}}{\operatorname{minimize}} & \sum_{t=0}^{N-1}\left\langle x_{t}, x_{t}\right\rangle+\left\langle u_{t}, u_{t}\right\rangle \\
\text { subject to } & \left\{\begin{array}{l}
\text { dynamics }(6.5), \\
x_{0}=\bar{x}, \\
u_{t} \in[0,1] \text { for } t=0, \ldots, N-1 .
\end{array}\right.
\end{array}
$$

If $\left(\left(x_{t}^{*}\right)_{t=0}^{N},\left(u_{t}^{*}\right)_{t=0}^{N-1}\right)$ is a solution of the problem (6.6), then Theorem 4.2 gives the following first order necessary conditions: 0 and

there exist $\eta_{c} \in\{0,1\}$ and a sequence of adjoint vectors $\left(\eta_{t}^{f}\right)_{t=0}^{N-1}$ such that $\left(\eta_{c},\left(\eta_{t}^{f}\right)_{t=0}^{N-1}\right) \neq$

- state and adjoint dynamics:

The function $f$ in (6.5) is differentiable everywhere on $\mathbb{R}^{2}$ except at a point $\left(x^{(1)}, x^{(2)}\right)=$ 

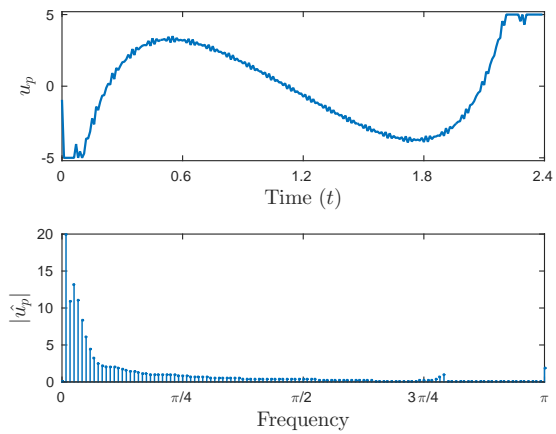

(A) Control corresponding to frequency constrained PMP: Observe, $u_{\mathrm{p}}$ does not voialate the control bound

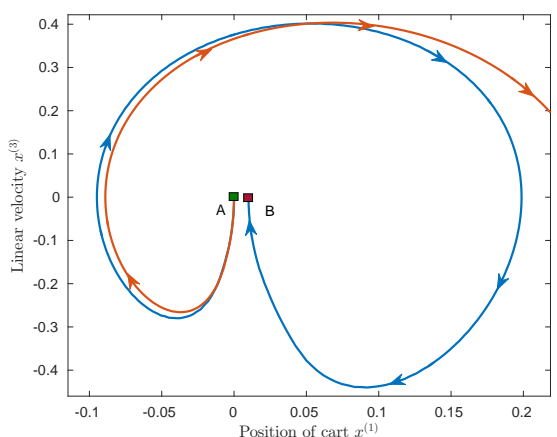

(c) Phase portraits of the linear position of cart vs its linear velocity
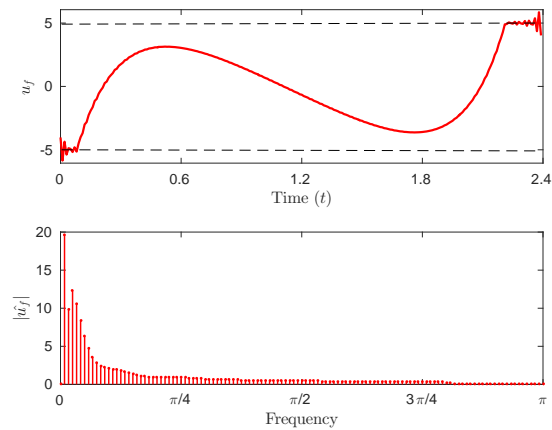

(в) Filtered control: Observe, at the begining and at the end of the trajectory $u_{\mathrm{f}}$ violates the control bound

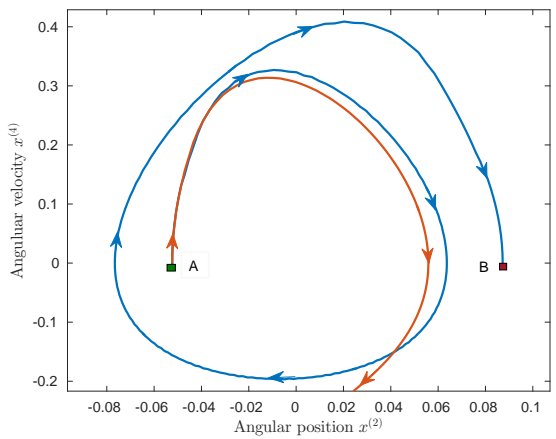

(D) Phase portraits of angular position of the pendulum vs its angular velocity

Figure 3. Time and frequency domain profiles of the optimal control $u_{\mathrm{p}}$ (obtained using the proposed necessary conditions) (Fig. 3A), and $u_{\mathrm{f}}$ (the filtered optimal control) (Fig. 3B). Phase portraits of the system for the initial condition $\bar{x}$ corresponding to the control inputs: $u_{\mathrm{p}}$ (in blue) and $u_{\mathrm{f}}$ (in red).

$(0,0)$. Therefore, the adjoint dynamics on $\mathbb{R}^{2} \backslash\{(0,0)\}$ is obtained from the condition (i) of the corollary 4.9 and the adjoint dynamics at $(0,0)$ is obtained from the condition (C-iii) of Theorem 4.2.

for $t=0, \ldots, N-1$,

$$
x_{t+1}^{*}=\left(\begin{array}{c}
x_{t}^{*(1)}\left(1-u_{t}^{*}\right) \\
\left\|x_{t}^{*}\right\| u_{t}^{*}
\end{array}\right)
$$

for $t=1, \ldots, N-1$,

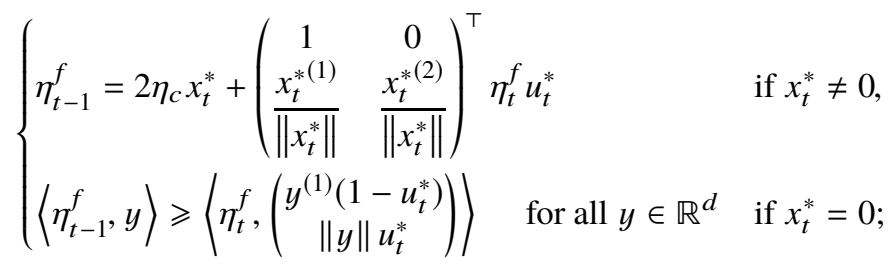


- transversality:

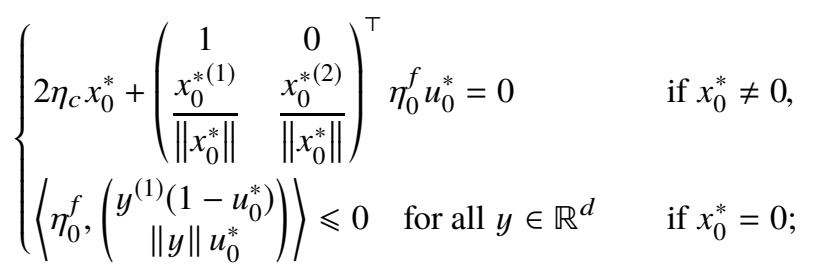

- Hamiltonian maximization:

The function governing dynamics of system (6.5) is smooth with respect to control variable $u$. Therefore the Hamiltonian maximization condition obtained using the condition $\left(\mathrm{v}^{*}\right)$ of the Corollary 4.9 is:

$$
2 \eta_{c} u_{t}^{*}=\left\langle\eta_{t}^{f},\left(\begin{array}{c}
-1 \\
\left\|x_{t}^{*}\right\|
\end{array}\right)\right\rangle .
$$

6.3. Example 3. Buck Converter: In $\S 6.2$ we have considered a system whose dynamics is smooth with respect to the control and nonsmooth with respect to the states. In the current subsection we consider a more general example where the system dynamics is nonsmooth with respect to the states as well as the control. The following difference equation represents modified discrete time buck converter system [BKYY00]. In particular, we suppose the clock cycle and the reference current are given and consider the voltage as our control input.

$$
i_{t+1}=f\left(i_{t}, v_{t}\right)= \begin{cases}\exp \left(\frac{-R T}{L}\right) i_{t}+\left(1-\exp \left(\frac{-R T}{L}\right)\right) \frac{v_{t}}{R} & \text { if } i_{t} \leqslant I_{\mathrm{b}}\left(v_{t}\right), \\ \left(\frac{v_{t}-i_{t} R}{v_{t}-I_{\mathrm{r}} R}\right)^{\left(\frac{R+R_{\mathrm{d}}}{R}\right)} I_{\mathrm{r}} \exp \left(-\frac{R+R_{\mathrm{d}}}{L} T\right) & \text { if } i_{t} \geqslant I_{\mathrm{b}}\left(v_{t}\right),\end{cases}
$$

where the different physical variables and parameters are as follows:

- $T$ is the time period of clock pulse,

$\circ i_{t}$ is the inductor current at $t^{t h}$ instant of time, and $v_{t}$ is the control input voltage,

- $R$ is the load resistance, $L$ is the inductance of inductor, and $R_{\mathrm{d}}$ is the diode resistance,

○ $I_{\mathrm{r}}$ is the reference current, $I_{\mathrm{b}}(v):=\left(I_{\mathrm{r}}-\frac{v}{R}\right) \exp \left(\frac{R T}{L}\right)+\frac{v}{R}$ is the borderline current at input voltage $v$, and $v_{\mathrm{b}}(i):=R\left(1-\exp \left(\frac{R T}{L}\right)\right)^{-1}\left(i-I_{\mathrm{r}} \exp \left(\frac{R T}{L}\right)\right)$ is the borderline voltagei when the current is $i$.

For more details on the dynamics, the reader may refer to [BKYY00]. To further compress the notation, we define

$$
\begin{aligned}
& \circ a:=\exp \left(\frac{-R T}{L}\right), b:=\frac{1-a}{R}, c:=R I_{\mathrm{r}} \exp \left(-\frac{\left(R+R_{\mathrm{d}}\right)}{L} T\right), \\
& \circ d:=I_{\mathrm{r}} \exp \left(-\frac{\left(R+R_{\mathrm{d}}\right)}{L} T\right), v_{\mathrm{r}}=I_{\mathrm{r}} R
\end{aligned}
$$

Assuming that the diode resistance is very small compared to the load resistance, $\frac{R+R_{\mathrm{d}}}{R} \approx$ 1 , the dynamics is represented in a compact form as

$$
i_{t+1}=f\left(i_{t}, v_{t}\right)= \begin{cases}a i_{t}+b v_{t} & \text { if } i_{t} \leqslant I_{\mathrm{b}}\left(v_{t}\right), \\ -c\left(\frac{i_{t}}{v_{t}-v_{\mathrm{r}}}\right)+d\left(\frac{v_{t}}{v_{t}-v_{\mathrm{r}}}\right) & \text { if } i_{t} \geqslant I_{\mathrm{b}}\left(v_{t}\right) .\end{cases}
$$

Observe that the map $\mathbb{R} \times \mathbb{R} \ni(i, v) \longmapsto f(i, v) \in \mathbb{R}$ in (6.12) governing the dynamics of the system is nonsmooth at $i=I_{\mathrm{b}}(v)$.

We consider the following optimal control problem where we minimize the power loss in the load and input voltage while transferring the system from a given initial state $i_{0}=\bar{i}$ 
to a given final state $i_{N}=i_{\mathrm{f}}$ in $N$ time steps.

$$
\begin{array}{ll}
\text { minimize } & \sum_{t=0}^{N-1} i_{t}^{2}+v_{t}^{2} \\
\text { subject to } & \left\{\begin{array}{l}
\text { dynamics (6.12) } \\
i_{0}=i_{i} \\
i_{N}=i_{f} .
\end{array}\right.
\end{array}
$$

If $\left(\left(i_{t}^{*}\right)_{t=0}^{N},\left(v_{t}^{*}\right)_{t=0}^{N-1}\right)$ is a solution of the problem (6.13), then the first order necessary conditions in Theorem 4.2 translate to the following conditions: there exist $\eta_{c} \in\{0,1\}$ and a sequence $\left(\eta_{t}^{f}\right)_{t=0}^{N-1}$, not all zero simultaneously, satisfying

- state and adjoint dynamics:

for $t=0, \ldots, N-1$,

$$
i_{t+1}^{*}= \begin{cases}a i_{t}^{*}+b v_{t}^{*} & \text { if } i_{t}^{*}<I_{\mathrm{b}}\left(v_{t}^{*}\right), \\ -c\left(\frac{i_{t}^{*}}{v_{t}^{*}-v_{\mathrm{r}}}\right)+d\left(\frac{v_{t}^{*}}{v_{t}^{*}-v_{\mathrm{r}}}\right) & \text { if } i_{t}^{*} \geqslant I_{\mathrm{b}}\left(v_{t}^{*}\right),\end{cases}
$$

for $t=1, \ldots, N-1$,

$$
\begin{cases}\eta_{t-1}^{f}=-2 \eta_{c} i_{t}^{*}-\eta_{t}^{x}+a \eta_{t}^{f} & \text { if } i_{t}^{*}<I_{\mathrm{b}}, \\ \eta_{t-1}^{f}=-2 \eta_{c} i_{t}^{*}-\eta_{t}^{x}-\frac{c}{v_{t}^{*}-v_{\mathrm{r}}} \eta_{t}^{f} & \text { if } i_{t}^{*}>I_{\mathrm{b}}\left(v_{t}^{*}\right), \\ -\frac{v_{\mathrm{r}} \eta_{t}^{f}}{v_{t}^{*}-v_{\mathrm{r}}} \leqslant \frac{\eta_{t-1}^{f}+\eta_{t}^{x}+2 \eta_{c} i_{t}^{*}}{a} \leqslant \eta_{t}^{f} & \text { if } i_{t}^{*}=I_{\mathrm{b}}\left(v_{t}^{*}\right) ;\end{cases}
$$

Notice that when $i_{t}^{*} \neq I_{\mathrm{b}}\left(v_{t}^{*}\right)$, the dynamics is smooth with respect to the states and the adjoint dynamics is given by the classical discrete time PMP and when $i_{t}^{*}=I_{\mathrm{b}}\left(v_{t}^{*}\right)$, the adjoint dynamics is given by the set inclusion in Theorem 4.2.

- transversality

$$
\begin{aligned}
& \left\{\begin{array}{ll}
2 \eta_{c} i_{0}^{*}+\eta_{0}^{x}=a \eta_{0}^{f} & \text { if } i_{t}^{*}<I_{\mathrm{b}}\left(v_{t}^{*}\right), \\
2 \eta_{c} i_{0}^{*}+\eta_{0}^{x}=-a \frac{v_{\mathrm{r}}}{v_{t}^{*}-v_{\mathrm{r}}} \eta_{0}^{f} & \text { if } i_{t}^{*}>I_{\mathrm{b}}\left(v_{t}^{*}\right), \\
-\frac{v_{\mathrm{r}} \eta_{0}^{f}}{v_{0}^{*}-v_{\mathrm{r}}} \leqslant \frac{\eta_{0}^{x}+2 \eta_{c} i_{0}^{*}}{a} \leqslant \eta_{0}^{f} & \text { if } i_{t}^{*}=I_{\mathrm{b}}\left(v_{t}^{*}\right),
\end{array}\right. \text { and } \\
& \eta_{N}^{f}=-\eta_{N}^{x} ;
\end{aligned}
$$

- Hamiltonian maximization

$$
\begin{cases}\eta_{c} v_{t}^{*}=\frac{c i_{t}^{*}+d v_{\mathrm{r}}}{2\left(v_{t}^{*}-v_{\mathrm{r}}\right)^{2}} \eta_{t}^{f} & \text { if } v_{t}^{*}<v_{\mathrm{b}}\left(i_{t}^{*}\right), \\ \eta_{c} v_{t}^{*}=\frac{b}{2} \eta_{t}^{f} & \text { if } v_{t}^{*}>v_{\mathrm{b}}\left(i_{t}^{*}\right), \\ b \eta_{t}^{f} \leqslant 2 \eta_{c} v_{t}^{*} \leqslant \frac{c i_{t}^{*}+d v_{\mathrm{r}}}{\left(v_{t}^{*}-v_{\mathrm{r}}\right)^{2}} \eta_{t}^{f} & \text { if } v_{t}^{*}=v_{\mathrm{b}}\left(i_{t}^{*}\right) .\end{cases}
$$

The dynamics is smooth with respect to the control when $v_{t}^{*} \neq v_{\mathrm{b}}\left(i_{t}^{*}\right)$ and hence the Hamiltonian maximization condition is same as the one given by Corollary 4.9. If 
$v_{t}^{*}=v_{\mathrm{b}}\left(i_{t}^{*}\right)$, then the system dynamics is not differentiable with respect to the control and the Hamiltonian maximization condition corresponds to a set inclusion.

The adjoint equation (6.8) and the transversality condition (6.9) for the problem (6.6), and the adjoint equation (6.16), the transversality condition (6.17) and the Hamiltonian maximization condition (6.18) for the problem (6.13) involve set-theoretic inclusion conditions. Most commercially available algorithms for solving two point boundary value problems involve equalities instead of inclusions, and can, therefore, not be applied directly to these problems. However, techniques based on semismooth Newtons methods (see, e.g., [Hin10]) appear to be promising directions for our problems; numerical algorithms based on these methods for synthesis of optimal control trajectories are under investigation and will be reported subsequently.

\section{Appendix A. Nonsmooth Optimization and Convex Cones}

Theorem A.1. [Cla13, Theorem 10.47 on p. 221] Consider an optimization problem

$$
\text { minimize } C(z)
$$

$$
\text { subject to }\left\{\begin{array}{l}
h(z)=0, \\
g(z) \leqslant 0, \\
z \in S,
\end{array}\right.
$$

where the functions governing cost, equality constraints, and inequality constraints are given by the maps $\mathbb{R}^{n} \ni z \longmapsto C(z) \in \mathbb{R}, \mathbb{R}^{n} \ni z \longmapsto h(z) \in \mathbb{R}^{v_{h}}$, and $\mathbb{R}^{n} \ni z \longmapsto g(z) \in$ $\mathbb{R}^{v_{g}}$ respectively, and $S$ is a closed subset of $\mathbb{R}^{n}$. If $z^{*}$ solves (A.1) and $C, h, g$ are Lipschitz near $z^{*}$, then there exist $\left(\eta_{c}, \lambda_{h}, \gamma_{g}\right) \in \mathbb{R} \times \mathbb{R}^{v_{h}} \times \mathbb{R}^{v_{g}}$ satisfying

(i) the nontriviality condition

$$
\left(\eta_{c}, \lambda_{h}, \gamma_{g}\right) \neq 0
$$

(ii) non-negativity and complementary slackness

$$
\eta_{c} \in\{0,1\}, \quad \gamma_{g} \geqslant 0, \quad\left\langle\gamma_{g}, g\left(z^{*}\right)\right\rangle=0
$$

(iii) and the stationarity condition

$$
0 \in \partial\left(\eta_{c} C+\left\langle\lambda_{h}, h\right\rangle+\left\langle\gamma_{g}, g\right\rangle\right)\left(z^{*}\right)+N_{S}^{C}\left(z^{*}\right) .
$$

Theorem A.2. [Bol75, Theorem 3 on p. 7] Let $K_{1}, \ldots, K_{s}$ be closed convex cones in $\mathbb{R}^{n}$ with vertex at 0 . If the cone $K=\operatorname{co}\left(\bigcup_{i=1}^{s} K_{i}\right)$ is not closed, then there are vectors $\lambda_{1} \in K_{1}, \ldots, \lambda_{s} \in K_{s}$, not all of them zero, such that $\sum_{i=1}^{s} \lambda_{i}=0$.

Theorem A.3. [Bol75, Theorem 4 on p. 8] Let $K_{1}, \ldots, K_{s}$ be closed convex cones in $\mathbb{R}^{d}$ with vertex at $x_{0}$. Then $\left(\bigcap_{i=1}^{s} K_{i}\right)^{+}=\operatorname{cl}\left(\operatorname{co}\left(\bigcup_{i=0}^{s} K_{i}^{+}\right)\right)$.

\section{Appendix B. Auxiliary Lemmas}

Lemma B.1. [Cla13, Chapter 10 on p. 201] Consider two functions $g_{1}, g_{2}: \mathbb{R}^{v} \longrightarrow \mathbb{R}$ such that in an open neighbourhood of $z \in \mathbb{R}^{v}, g_{1}$ is Lipschitz continuous and $g_{2}$ is continuously differentiable. If $\partial g(\cdot)$ denotes the generalized differential then,

$$
\partial\left(g_{1}+g_{2}\right)(z)=\partial g_{1}(z)+\frac{\partial}{\partial z} g_{2}(z) .
$$

Lemma B.2. Let $N$ be a positive integer, $S_{i} \subset \mathbb{R}^{m}$ be closed and nonempty sets for $i=1, \ldots, N$. Then $T_{S}^{C}\left(z^{*}\right)=\bigcap_{i=1}^{N} T_{S_{i}}^{C}\left(z^{*}\right)$ for $z^{*} \in S:=\bigcap_{i=1}^{N} S_{i}$, 
Proof. For $v \in T_{S}^{C}\left(z^{*}\right), d_{S}^{\circ}\left(z^{*} ; v\right)=0$. Since $S \subset S_{i}, d_{S_{i}}^{\circ}\left(z^{*} ; v\right)=0$ for each $i=1, \ldots, N$ and hence $v \in\left(\bigcap_{i=1}^{N} T_{S_{i}}^{C}\left(z^{*}\right)\right)$. Conversely, if $v \in \bigcap_{i=1}^{N} T_{S_{i}}^{C}\left(z^{*}\right)$, then $d_{S_{i}}^{\circ}\left(z^{*} ; v\right)=0$ for $i=$ $1, \ldots, N$. Thus, $d_{S}^{\circ}\left(z^{*} ; v\right)=0$ and $v \in T_{S}^{C}\left(z^{*}\right)$.

\section{REFERENCES}

[AB08] V. Acary and B. Brogliato, Numerical Methods for Nonsmooth Dynamical Systems: Applications in Mechanics and Electronics, vol. 35, Springer, New York, 2008.

[ABSP11] J.-P. Aubin, A. M. Bayen, and P. Saint-Pierre, Viability Theory: New Directions, 2 ed., Springer, Berlin, 2011.

[Ber12] D. P. Bertsekas, Dynamic Programming and Optimal Control, Vol. I, Athena Scientific, Belmont, MA, 2012.

[BKYY00] S. Banerjee, M. S. Karthik, G. Yuan, and J. A. Yorke, Bifurcations in one-dimensional piecewise smooth maps-theory and applications in switching circuits, IEEE Transactions on Circuits and Systems I: Fundamental Theory and Applications 47 (2000), no. 3, 389-394.

[Bol75] V. G. Boltyanskii, The method of tents in the theory of extremal problems, Russian Mathematical Surveys 30 (1975), no. 3, 1-54.

[Cla13] F. H. Clarke, Functional Analysis, Calculus of Variations and Optimal Control, Graduate Texts in Mathematics, vol. 264, Springer, London, 2013.

[CLSW98] F. H. Clarke, Y. S. Ledyaev, R. J. Stern, and P. R. Wolenski, Nonsmooth Analysis and Control Theory, Graduate Texts in Mathematics, vol. 178, Springer-Verlag, New York, 1998.

[DFT92] J. C. Doyle, B. A. Francis, and A. R. Tannenbaum, Feedback Control Theory, Macmillan Publishing Company, New York, 1992.

[DM65] A. Ya. Dubovitskii and A. A. Milyutin, Extremum problems in the presence of restrictions, USSR Computational Mathematics and Mathematical Physics 5 (1965), no. 3, 1-80.

[Dub78] A. Ya. Dubovitskiū, The discrete maximum principle (Russian), Akademiya Nauk SSSR. Avtomatika i Telemekhanika (1978), no. 10, 55-71.

[GP17] L. Grüne and J. Pannek, Nonlinear Model Predictive Control: Theory and Algorithms, 2nd ed., Communication and Control Engineering Series, Springer International Publishing Switzerland, 2017.

[Gül10] O. Güler, Foundations of Optimization, Graduate Texts in Mathematics, vol. 258, Springer, New York, 2010.

[Hin10] M. Hintermüller, Semismooth Newton Methods and Applications, https://www . math.uni-hamburg.de/home/hinze/Psfiles/Hintermueller_owNotes.pdf, Oberwolfach-Seminar on "Mathematics of PDE constrained Optimization" at Mathematische Forschungsintitut in Oberwolfach, 2010.

[HUL93] J.-B. Hiriart-Urruty and C. Lemaréchal, Convex Analysis and Minimization Algorithms I, Grundlehren der Mathematischen Wissenschaften [Fundamental Principles of Mathematical Sciences], SpringerVerlag, Berlin, 1993, Fundamentals.

[IT09] A. D. Ioffe and V. M. Tihomirov, Theory of Extremal Problems, Studies in Mathematics and its Applications, North-Holland Publishing Company, Amsterdam, 2009.

$\left[\mathrm{KPP}^{+} 18\right]$ S. Kotpalliwar, P. Paruchuri, K. S. Phogat, D. Chatterjee, and R. N. Banavar, A frequency-constrained geometric Pontryagin maximum principle on matrix Lie groups, 2018, http: //arxiv.org/abs/1803.03052.

[Lib11] D. Liberzon, Calculus of Variations and Optimal Control Theory: A Concise Introduction, Princeton University Press, 2011.

[Mor06a] B. S. Mordukhovich, Variational Analysis and Generalized Differentiation I, Grundlehren der Mathematischen Wissenschaften [Fundamental Principles of Mathematical Sciences], Springer-Verlag, Berlin, 2006, Applications.

[Mor06b] - Variational Analysis and Generalized Differentiation II, Grundlehren der Mathematischen Wissenschaften [Fundamental Principles of Mathematical Sciences], Springer-Verlag, Berlin, 2006, Applications.

[MS04] B. S. Mordukhovich and I. Shvartsman, The approximate maximum principle in constrained optimal control, SIAM Journal on Control and Optimization 43 (2004), no. 3, 1037-1062.

[PC17] P. Paruchuri and D. Chatterjee, Discrete time Pontryagin maximum principle for optimal control problems under state-action-frequency constraints, 2017, https://arxiv .org/abs/1708.04419.

[PC19] Discrete time Pontryagin maximum principle for optimal control problems under state-action-frequency constraints, IEEE Transactions on Automatic Control (2019), doi: https://doi.org/10.1109/TAC.2019.2893160; extended version available at https://arxiv.org/abs/1708.04419.

[Pše71] B. N. Pšeničny̆, Necessary Conditions for an Extremum, Marcel Dekker Inc., New York, 1971. 
[SB95] J. A. Scaramuzzo and R. W. Brockett, Band limited control of a flexible structure using piecewise trigonometric input signals, 07 Nov 1995, US Patent 5465035 A.

[SS03] E. M. Stein and R. Shakarchi, Fourier Analysis, Princeton Lectures in Analysis, vol. 1, Princeton University Press, Princeton, NJ, 2003, An introduction.

[SW97] H. J. Sussmann and J. C. Willems, 300 years of optimal control: from the brachystochrone to the maximum principle, IEEE Control Systems Magazines, June 1997, pp. 32-44. 